\title{
A consistent description of kinetics and hydrodynamics of quantum Bose-systems
}

\author{
P.A.Hlushak, M.V.Tokarchuk \\ Institute for Condensed Matter Physics \\ of the National Academy of Sciences of Ukraine, \\ 1 Svientsitskii Str., 79011 Lviv, Ukraine
}

Received August 8, 2004

\begin{abstract}
A consistent approach to the description of kinetics and hydrodynamics of many-Boson systems is proposed. The generalized transport equations for strongly and weakly nonequilibrium Bose systems are obtained. Here we use the method of nonequilibrium statistical operator by D.N. Zubarev. New equations for the time distribution function of the quantum Bose system with a separate contribution from both the kinetic and potential energies of particle interactions are obtained. The generalized transport coefficients are determined accounting for the consistent description of kinetic and hydrodynamic processes.
\end{abstract}

Key words: Bose system, helium, kinetics, hydrodynamics, correlation function, transport coefficients

PACS: $67.40 .-w, 47.37 .+q$

\section{Introduction}

The theoretical investigation of nonequilibrium properties of vapour helium and their change at transition with the decrease of the temperature lower than $T_{\mathrm{c}}=4.2 \mathrm{~K}$ in a fluid state $\mathrm{HeI}$, and lower than $T_{\lambda}=2.17 \mathrm{~K}$ in a liquid state HeII that is characterized by superfluidity, remains an urgent issue in the modern statistical theory of nonequilibrium processes of quantum systems. To construct a nonequilibrium statistical theory capable of consistensly describing vapour, liquid and superfluid helium in view of phase transitions is a real problem for every theorist interested in the unique physical properties of helium. The quantum system of Bose particles serves as a physical model in theoretical descriptions of both the equilibrium and nonequilibrium properties of real helium. In particular, many articles [1-16] are devoted to the hydrodynamic description of normal and superfluid states of such a system. A brief review of the results of investigations within the framework of linear hydrodynamics 
has been carried out in an article by Tserkovnikov [16]. In papers [17-19] theoretical approaches are proposed to the description of nonlinear hydrodynamic fluctuations connected with the problem of calculating the dispersion for the kinetic transport coefficients and a spectrum of collective modes in the low-frequency area for a superfluid Bose liquid. Problems of building the kinetic equation for Bose systems based on the microscopic approach were considered in papers [20,21]. For normal Bose systems, the calculations of the collective mode spectrum (without accounting for a thermal mode), dynamic structure factor, kinetic transport coefficients $[9$, see the reference] are carried out based on the hydrodynamic or kinetic approaches. Nevertheless, these results are valid only in the hydrodynamic area (i.e., small values of wave vector $\mathbf{k}$ and frequency $\omega$ ). For superfluid helium, some papers [22-24] were devoted to the investigation of the dynamic structure factor and spectrum of collective excitations.

In papers $[25,26]$, a generalized scheme for theoretical description of dynamic properties of semiquantum helium has been proposed based on the method of nonequilibrium statistical operator. Here the set of equations of generalized hydrodynamics is obtained and the thermal viscous model with kinetic and hydrodynamical collective modes is analyzed in detail. The closed system of the equations for time correlation functions is obtained using the Markovian approximation for transport kernels. Using these equations the analysis of dynamic properties of semiquantum helium is carried out at two values of temperature above the transition to a superfluid state. Similar investigations were performed in papers [27-29] for helium above the point of the phase transition.

In general, there exists a hard problem in describing the Bose systems going out from the hydrodynamic area to the area of intermediate values of $\mathbf{k}$ and $\omega$, where the kinetic and hydrodynamic processes are interdependent and should be considered simultaneously. This is one of the urgent problems in the statistical theory of nonequilibrium processes of transport in a quantum liquid. It should be noted that in the paper by J.O.Tserkovnikov [30], a problem of building the linearized kinetic equation for the Bose-system above critical temperature was considered using the method of two-time Green functions [31,32].

The main step in this direction has been made when investigating semiquantum helium $[25,26]$. A considerable success was achieved in papers [33-36] in which the approach to the consistent description of kinetics and hydrodynamics of classical dense gases and fluids is proposed based on the method of nonequilibrium statistical operator by D.N.Zubarev $[37,38]$. In the present study, we apply this approach to a consistent description of kinetics and hydrodynamics of many-particle Bose systems.

In the second part of the paper we shall obtain a nonequilibrium statistical operator of the system at the consistent description of kinetics and hydrodynamics using the method of the nonequilibrium statistical operator. The quantum nonequilibrium one-particle distribution function and the average value of the potential energy of interaction (for which the closed system of the transport equations is obtained) have been selected as parameters of this consistent description of a nonequilibrium state.

In the third part, the kinetics and hydrodynamics of weakly nonequilibrium 
Bose gas are considered. Here the self-consistent transport equations are obtained for parameters of an abbreviated description based on their solutions, the kinetic equation for the quantum one-partial distribution function is written in a form where the transport kernels contain a renormalization of kinetical correlations by hydrodynamical ones. Moreover, we also obtain the system of equations for time correlation functions of parameters of a consistent description. From these equations, the dynamic structure factor of the system as well as the time correlation functions related to momentum and energy fluctuations are determined.

\section{The nonequilibrium statistical operator for Bose system}

Let us consider a normal Bose system with the Hamiltonian

$$
\hat{H}=\sum_{\mathbf{k}} \sum_{\mathbf{p}} \frac{p^{2}}{2 m} \hat{a}_{\mathbf{p}-\frac{\mathbf{k}}{2}}^{+} \hat{a}_{\mathbf{p}+\frac{\mathbf{k}}{2}}+\frac{1}{2 V} \sum_{\mathbf{k}} \sum_{\mathbf{q}} \sum_{\mathbf{p}} \nu(q) \hat{a}_{\mathbf{p}+\frac{\mathbf{q}-\mathbf{k}}{2}}^{+} \hat{\rho}_{\mathbf{q}} \hat{a}_{\mathbf{p}-\frac{\mathbf{q}-\mathbf{k}}{2}},
$$

where $\hat{a}_{\mathbf{p}}$ and $\hat{a}_{\mathbf{p}}^{+}$are the Bose operators of annihilation and creation of particles in the state with momentum $\mathbf{p}, \nu(q)=\int \exp (-\mathbf{i q r}) \Phi(|\mathbf{r}|) \mathrm{d} \mathbf{r}$ is the Fourier-component of the interaction potential between particles, $V$ is the volume,

$$
\hat{\rho}_{\mathbf{q}}=\frac{1}{\sqrt{N}} \sum_{\mathbf{p}} \hat{a}_{\mathbf{p}-\frac{\mathbf{q}}{2}}^{+} \hat{a}_{\mathbf{p}+\frac{\mathbf{q}}{2}}
$$

is the Fourier-component of the operator of number particles density, $N$ is the total number of particles.

The nonequilibrium state of such a quantum system is completely described by the nonequilibrium statistical operator $\hat{\rho}(t)$ which satisfies the quantum Liouville equation:

$$
\frac{\partial}{\partial t} \hat{\rho}(t)+\mathrm{i} \hat{L}_{N} \hat{\rho}(t)=0
$$

where Liouville operator $\mathrm{i} \hat{L}_{N} \hat{A}=\mathrm{i} / \hbar \cdot[\hat{A}, \hat{H}]$. To solve the equation it is necessary to set initial conditions. We shall use the method of nonequilibrium statistical operator $[37,38]$. From the very beginning we consider the problem of selecting such solutions to the equation (2.3) which correspond to the ideas of abbreviated description [38]. These solutions depend on time only through average values of the set of observable quantities $\left\langle\hat{P}_{m}\right\rangle^{t}$ and do not depend on the initial moment of time $t_{0}$ : $\hat{\rho}(t)=\hat{\rho}\left(\ldots\left\langle\hat{P}_{m}\right\rangle^{t} \ldots\right)$. Such solutions can be obtained by including an infinitesimal source in the right-hand side of Liouville equation $(2.3)[37,38]$ :

$$
\frac{\partial}{\partial t} \hat{\rho}(t)+\mathrm{i} \hat{L}_{N} \hat{\rho}(t)=-\epsilon\left(\hat{\rho}(t)-\hat{\rho}_{\mathrm{q}}(t)\right)
$$

where $\epsilon \rightarrow+0$ after the limiting thermodynamic transition. The sourse breaks the symmetry of the equation with respect to $t \rightarrow-t$ and selects retarded solutions which correspond to an abbreviated description of the nonequilibrium system. The 
quasiequilibrium statistical operator $\hat{\rho}_{\mathrm{q}}(t)$ is defined from the condition of an extreme of the information entropy of the system at the conservation of the normalization condition

$$
\operatorname{Sp} \hat{\rho}_{\mathrm{q}}(t)=1
$$

and at fixed values of the quantities $\left\langle\hat{P}_{m}\right\rangle^{t}$ (parameters of an abbreviated description).

When investigating a hydrodynamical nonequilibrium state of the normal Bose liquid, which is characterized by transport of energy, momentum and mass, the observable quantities such as the average values of energy density $\left\langle\hat{\varepsilon}_{\mathbf{q}}\right\rangle^{t}$, momentum $\left\langle\hat{P}_{\mathbf{q}}\right\rangle^{t}$, and particle number $\left\langle\hat{\rho}_{\mathbf{q}}\right\rangle^{t}[10,14,16]$ are chosen as the parameters of an abbreviated description. The averaging is defined as

$$
\langle(\ldots)\rangle^{t}=\operatorname{Sp}[(\ldots) \hat{\rho}(t)]
$$

The Fourier-components of the energy density and momentum density

$$
\begin{aligned}
\hat{\varepsilon}_{\mathbf{q}} & =\frac{1}{\sqrt{N}} \sum_{\mathbf{p}}\left(\frac{p^{2}}{2 m}-\frac{q^{2}}{8 m}\right) \hat{a}_{\mathbf{p}-\frac{\mathbf{q}}{2}}^{+} \hat{a}_{\mathbf{p}+\frac{\mathbf{q}}{2}}+\frac{1}{2 V} \sum_{\mathbf{p}} \sum_{\mathbf{k}} \nu(k) \hat{a}_{\mathbf{p}+\frac{\mathbf{q}-\mathbf{k}}{2}} \hat{\rho}_{\mathbf{k}} \hat{a}_{\mathbf{p}-\frac{\mathbf{q}-\mathbf{k}}{2}}, \\
\hat{\mathbf{P}}_{\mathbf{q}} & =\frac{1}{\sqrt{N}} \sum_{\mathbf{p}} \mathbf{p} \hat{a}_{\mathbf{p}-\frac{\mathbf{q}}{2}}^{+} \hat{a}_{\mathbf{p}+\frac{\mathbf{q}}{2}}
\end{aligned}
$$

together with the number of particles density (2.2) satisfy local conservation laws. Their averaged values satisfy the equations of the generalized hydrodynamics of quantum systems (in the linear approximation of the equations of the molecular hydrodynamics). The important feature of the densities of energy, momentum and number of particles, which is locally conserved, is that they are defined through the Klimontovich operator of the phase particles number density $\hat{n}_{\mathbf{q}}(\mathbf{p})$ :

$$
\begin{aligned}
\hat{n}_{\mathbf{q}}(\mathbf{p}) & =\hat{a}_{\mathbf{p}-\frac{\mathbf{q}}{2}}^{+} \hat{a}_{\mathbf{p}+\frac{\mathbf{q}}{2}}, \\
\hat{\rho}_{\mathbf{q}} & =\frac{1}{\sqrt{N}} \sum_{\mathbf{p}} \hat{n}_{\mathbf{q}}(\mathbf{p}), \\
\hat{\mathbf{P}}_{\mathbf{q}} & =\frac{1}{\sqrt{N}} \sum_{\mathbf{p}} \mathbf{p} \hat{n}_{\mathbf{q}}(\mathbf{p}), \\
\hat{\varepsilon}_{\mathbf{q}}^{\text {kin }} & =\frac{1}{\sqrt{N}} \sum_{\mathbf{p}}\left(\frac{p^{2}}{2 m}-\frac{q^{2}}{8 m}\right) \hat{n}_{\mathbf{q}}(\mathbf{p}), \\
\hat{\varepsilon}_{\mathbf{q}}^{\text {int }} & =\frac{1}{2 V} \sum_{\mathbf{p}} \sum_{\mathbf{p}^{\prime}} \sum_{\mathbf{k}} \nu(k) \hat{a}_{\mathbf{p}+\frac{\mathbf{k}-\mathbf{q}}{2}}^{+} \hat{n}_{\mathbf{k}}\left(p^{\prime}\right) \hat{a}_{\mathbf{p}-\frac{\mathbf{k}-\mathbf{q}}{2}},
\end{aligned}
$$

where $\hat{\varepsilon}_{\mathbf{q}}^{\text {kin }}$ and $\hat{\varepsilon}_{\mathbf{q}}^{\text {int }}$ are the Fourier-components of operators of kinetic and potential energy density. The averaged values for these quantities can be written as:

$$
\left\langle\hat{\rho}_{\mathbf{q}}\right\rangle^{t}=\frac{1}{\sqrt{N}} \sum_{\mathbf{p}} f_{1}(\mathbf{p}, \mathbf{q}, t),
$$




$$
\begin{aligned}
\left\langle\hat{\mathbf{P}}_{\mathbf{q}}\right\rangle^{t} & =\frac{1}{\sqrt{N}} \sum_{\mathbf{p}} \mathbf{p} f_{1}(\mathbf{p}, \mathbf{q}, t), \\
\left\langle\hat{\varepsilon}_{\mathbf{q}}^{\text {kin }}\right\rangle^{t} & =\frac{1}{\sqrt{N}} \sum_{\mathbf{p}}\left(\frac{p^{2}}{2 m}-\frac{q^{2}}{8 m}\right) f_{1}(\mathbf{p}, \mathbf{q}, t) .
\end{aligned}
$$

The one-particle distribution function $f(\mathbf{p}, \mathbf{q}, t)=\left\langle\hat{n}_{\mathbf{q}}(\mathbf{p})\right\rangle^{t}$ satisfies the kinetic equation for the quantum Bose system. On the other hand (2.14)-(2.16), the average value of the potential energy is defined through the quantum two-particle nonequilibrium distribution function:

$$
\left\langle\hat{\varepsilon}_{\mathbf{q}}^{\text {int }}\right\rangle^{t}=\frac{1}{2 V} \sum_{\mathbf{p}} \sum_{\mathbf{p}^{\prime}} \sum_{\mathbf{k}} \nu(k) f_{2}\left(\mathbf{p}+\frac{\mathbf{k}-\mathbf{q}}{2} ; \mathbf{p}^{\prime} ; \mathbf{p}-\frac{\mathbf{k}-\mathbf{q}}{2}, t\right),
$$

where

$$
f_{2}\left(\mathbf{p}+\frac{\mathbf{k}-\mathbf{q}}{2} ; \mathbf{p}^{\prime} ; \mathbf{p}-\frac{\mathbf{k}-\mathbf{q}}{2} ; t\right)=\left\langle\hat{a}_{\mathbf{p}+\frac{\mathbf{k}-\mathbf{q}}{2}}^{+} \hat{n}_{\mathbf{k}}\left(\mathbf{p}^{\prime}\right) \hat{a}_{\mathbf{p}-\frac{\mathbf{k}-\mathbf{q}}{2}}\right\rangle^{t}
$$

At this stage there is a problem of a consistent description of kinetics and hydrodynamics of the quantum Bose system. For the hydrodynamical description of a nonequilibrium state of the system it is enough to include in the set of parameters of an abbreviated description the average values of particle number $\left\langle\hat{\rho}_{\mathbf{q}}\right\rangle^{t}$, momentum $\left\langle\hat{\mathbf{P}}_{\mathbf{q}}\right\rangle^{t}$ and full energy $\left\langle\hat{\varepsilon}_{\mathbf{q}}\right\rangle^{t}$. On the other hand, the quantum one-particle distribution function, which satisfies the kinetic equation, is a characteristic parameter for the kinetic description of the nonequilibrium state of the system. The agreement between kinetics and hydrodynamics for very dilute Bose gas does not cause any problem because in this case the density is a small parameter. Therefore, only the quantum one-particle distribution function $f_{1}(\mathbf{p}, \mathbf{q}, t)$ can be chosen for a parameter of an abbreviated description.

At transition to quantum Bose liquids, the contribution of collective correlations, which are described by average potential energy of interaction $\left\langle\hat{\varepsilon}_{\mathbf{q}}^{\text {int }}\right\rangle^{t}(2.17)$, is more important than the one-particle correlations connected with $f_{1}(\mathbf{p}, \mathbf{q}, t)$. From this fact it follows that for a consistent description of kinetics and hydrodynamics of a Bose liquid, the quantum one-particle nonequilibrium distribution function $f_{1}(\mathbf{p}, \mathbf{q}, t)$ and the average potential energy of interaction $\left\langle\hat{\varepsilon}_{\mathbf{q}}^{\text {int }}\right\rangle^{t}$ are indispensable in order to be choosen as parameters of an abbreviated description for a nonequilibrium state. Similar problems of the consistent description of kinetics and hydrodynamics of classical dense gases and liquids, as was already noted above, were considered in papers [33-35]. Therefore, using papers [33,35,36,44], we shall find the quasiequilibrium statistical operator, which has been entered in (2.4), from the condition of the extremum of information entropy at the conservation of normalization condition (2.5) for fixed values of $\left\langle\hat{n}_{\mathbf{q}}(\mathbf{p})\right\rangle^{t}=f_{1}(\mathbf{p}, \mathbf{q}, t)$ and $\left.\left\langle\hat{\varepsilon}_{\mathbf{q}}\right\rangle^{t}\right\rangle^{t}$ :

$$
\hat{\rho}_{\mathrm{q}}(t)=\exp \left\{-\Phi(t)-\sum_{\mathbf{q}} \beta_{-\mathbf{q}}(t) \hat{\varepsilon}_{\mathbf{q}}^{\mathrm{int}}-\sum_{\mathbf{q}} \sum_{\mathbf{p}} \gamma_{-\mathbf{q}}(\mathbf{p} ; t) \hat{n}_{\mathbf{q}}(\mathbf{p})\right\} .
$$


The Lagrangian multipliers $\beta_{-\mathbf{q}}(t), \gamma_{-\mathbf{q}}(\mathbf{p} ; t)$ are defined from the self-consistent conditions:

$$
\begin{aligned}
\left\langle\hat{\varepsilon}_{\mathbf{q}}^{\text {int }}\right\rangle^{t} & =\left\langle\hat{\varepsilon}_{\mathbf{q}}^{\text {int }}\right\rangle_{\mathrm{q}}^{t}, \quad\left\langle\hat{n}_{\mathbf{q}}(\mathbf{p})\right\rangle^{t}=\left\langle\hat{n}_{\mathbf{q}}(\mathbf{p})\right\rangle_{\mathrm{q}}^{t}, \\
\langle(\ldots)\rangle_{\mathrm{q}}^{t} & =\operatorname{Sp}\left[(\ldots) \rho_{\mathrm{q}}(t)\right] .
\end{aligned}
$$

The Massieu-Planck functional

$$
\Phi(t)=\ln \operatorname{Sp} \exp \left\{-\sum_{\mathbf{q}} \beta_{-\mathbf{q}}(t) \hat{\varepsilon}_{\mathbf{q}}^{\mathrm{int}}-\sum_{\mathbf{q}} \sum_{\mathbf{p}} \gamma_{-\mathbf{q}}(\mathbf{p} ; t) \hat{n}_{\mathbf{q}}(\mathbf{p})\right\}
$$

is determined from the normalization condition (2.5).

At the given quasiequilibrium operator $\hat{\rho}_{\mathrm{q}}(t)(2.19)$ we shall find the nonequilibrium statistical operator $\hat{\rho}(t)$ that satisfies the quantum Liouville equation it the presence of a source. For this purpose we shall write down the equation (2.4) as [38]:

$$
\left[\frac{\partial}{\partial t}+\left(1-P_{\mathrm{q}}(t)\right) \mathrm{i} \hat{L}_{N}+\epsilon\right]\left(\hat{\rho}(t)-\hat{\rho}_{\mathrm{q}}(t)\right)=-\left(1-P_{\mathrm{q}}(t)\right) \mathrm{i} \hat{L}_{N} \hat{\rho}_{\mathrm{q}}(t),
$$

where the generalized Kawasaki-Gunton projection operator acts only on statistical operators

$$
\begin{aligned}
P_{\mathrm{q}}(t) \hat{\rho}^{\prime}= & {\left[\hat{\rho}_{\mathrm{q}}(t)-\sum_{\mathbf{q}} \frac{\delta \hat{\rho}_{\mathbf{q}}(t)}{\delta\left\langle\hat{\varepsilon}_{\mathbf{q}}^{\text {int }}\right\rangle^{t}}\left\langle\hat{\varepsilon}_{\mathbf{q}}^{\text {int }}\right\rangle^{t}-\sum_{\mathbf{q}} \sum_{\mathbf{p}} \frac{\delta \hat{\rho}_{\mathbf{q}}(t)}{\delta\left\langle\hat{n}_{\mathbf{q}}(\mathbf{p})\right\rangle^{t}}\left\langle\hat{n}_{\mathbf{q}}(\mathbf{p})\right\rangle^{t}\right] \operatorname{Sp} \hat{\rho}^{\prime} } \\
& +\sum_{\mathbf{q}} \frac{\delta \hat{\rho}_{\mathbf{q}}(t)}{\delta\left\langle\hat{\varepsilon}_{\mathbf{q}}^{\text {int }}\right\rangle^{t}} \operatorname{Sp}\left(\hat{\varepsilon}_{\mathbf{q}}^{\text {int }} \hat{\rho}^{\prime}\right)+\sum_{\mathbf{q}} \sum_{\mathbf{p}} \frac{\delta \hat{\rho}_{\mathbf{q}}(t)}{\delta\left\langle\hat{n}_{\mathbf{q}}(\mathbf{p})\right\rangle^{t}} \operatorname{Sp}\left(\hat{n}_{\mathbf{q}}(\mathbf{p}) \hat{\rho}^{\prime}\right)
\end{aligned}
$$

and has the following properties:

$$
P_{\mathrm{q}}(t) \hat{\rho}^{\prime}=\hat{\rho}_{\mathrm{q}}(t), \quad P_{\mathrm{q}}(t) \hat{\rho}_{\mathrm{q}}^{\prime}\left(t^{\prime}\right)=\hat{\rho}_{\mathrm{q}}(t), \quad P_{\mathrm{q}}(t) P_{\mathrm{q}}\left(t^{\prime}\right)=P_{\mathrm{q}}(t) .
$$

The formal solution of the equation (2.22) is of the form:

$$
\hat{\rho}(t)=\hat{\rho}_{\mathrm{q}}(t)-\int_{-\infty}^{t} \mathrm{~d} t^{\prime} \exp \left\{\epsilon\left(t^{\prime}-t\right)\right\} T_{\mathrm{q}}\left(t, t^{\prime}\right)\left(1-P_{\mathrm{q}}\left(t^{\prime}\right)\right) \mathrm{i} \hat{L}_{N} \hat{\rho}_{\mathrm{q}}\left(t^{\prime}\right),
$$

where

$$
T_{\mathrm{q}}\left(t, t^{\prime}\right)=\exp _{+}\left\{-\int_{t^{\prime}}^{t} \mathrm{~d} t^{\prime}\left(1-P_{\mathrm{q}}\left(t^{\prime}\right)\right) \mathrm{i} \hat{L}_{N}\right\}
$$

is the generalized evolution operator which takes into account the projection. Further, we shall act by operators $\left(1-P_{\mathrm{q}}\left(t^{\prime}\right)\right) \mathrm{i} \hat{L}_{N}$ on $\hat{\rho}_{\mathrm{q}}\left(t^{\prime}\right)$ in the right-hand side of (2.24). As a result, we obtain

$$
\begin{aligned}
\left(1-P_{\mathbf{q}}\left(t^{\prime}\right)\right) i \hat{L}_{N} \hat{\rho}_{\mathrm{q}}\left(t^{\prime}\right)=-\int_{0}^{1} \mathrm{~d} & \tau\left(\hat{\rho}_{\mathbf{q}}(t)\right)^{\tau}\left\{\sum_{\mathbf{q}} \beta_{-\mathbf{q}}(t) I_{\varepsilon}^{\mathrm{int}}(\mathbf{q}, t)\right. \\
& \left.+\sum_{\mathbf{q}} \sum_{\mathbf{q}} \gamma_{-\mathbf{q p}}(t) I_{n}(\mathbf{p}, \mathbf{q}, t)\right\}\left(\hat{\rho}_{\mathbf{q}}(t)\right)^{1-\tau},
\end{aligned}
$$


where the generalized flows are:

$$
\begin{aligned}
I_{\varepsilon}^{\mathrm{int}}(\mathbf{q}, t) & =(1-P(t)) \mathrm{i} \hat{L}_{N} \hat{\varepsilon}_{\mathbf{q}}^{\mathrm{int}} \\
I_{n}(\mathbf{p}, \mathbf{q}, t) & =(1-P(t)) \mathrm{i} \hat{L}_{N} \hat{n}_{\mathbf{q}}^{\mathrm{int}}(\mathbf{p}) .
\end{aligned}
$$

Expressions (2.27) and (2.28) contain the generalized Mori projection operator:

$$
P(t) \hat{b}=\langle\hat{b}\rangle_{\mathbf{q}}^{t}+\sum_{\mathbf{q}} \frac{\delta\langle\hat{b}\rangle_{\mathbf{q}}^{t}}{\delta\left\langle\hat{\varepsilon}_{\mathbf{q}}^{\text {int }}\right\rangle^{t}}\left(\hat{\varepsilon}_{\mathbf{q}}^{\mathrm{int}}-\left\langle\hat{\varepsilon}_{\mathbf{q}}^{\mathrm{int}}\right\rangle^{t}\right)+\sum_{\mathbf{q}} \sum_{\mathbf{p}} \frac{\delta\langle\hat{b}\rangle_{\mathbf{q}}^{t}}{\delta\left\langle\hat{n}_{\mathbf{q}}(\mathbf{p})\right\rangle^{t}}\left(\hat{n}_{\mathbf{q}}(\mathbf{p})-\left\langle\hat{n}_{\mathbf{q}}(\mathbf{p})\right\rangle^{t}\right),
$$

which acts only on operators of physical quantities and has the properties:

$$
P(t) \hat{n}_{\mathbf{q}}(\mathbf{p})=\hat{n}_{\mathbf{q}}(\mathbf{p}), \quad P(t) \hat{\varepsilon}_{\mathbf{q}}^{\text {int }}=\hat{\varepsilon}_{\mathbf{q}}^{\text {int }}, \quad P(t) P\left(t^{\prime}\right)=P(t) .
$$

Let us substitute (2.26) in (2.24). Then for the nonequilibrium statistical operator of Bose system we shall write:

$$
\begin{aligned}
\hat{\rho}(t)= & \rho_{\mathrm{q}}(t)+\sum_{\mathbf{q}} \int_{-\infty}^{t} \mathrm{~d} t^{\prime} \exp \left\{\epsilon\left(t^{\prime}-t\right)\right\} T_{\mathrm{q}}\left(t, t^{\prime}\right) \\
& \times \int_{0}^{1} \mathrm{~d} \tau\left(\rho_{\mathrm{q}}\left(t^{\prime}\right)\right)^{\tau} I_{\varepsilon}^{\mathrm{int}}\left(\mathbf{q}, t^{\prime}\right)\left(\rho_{\mathrm{q}}\left(t^{\prime}\right)\right)^{1-\tau} \beta_{-\mathbf{q}}\left(t^{\prime}\right) \\
& +\sum_{\mathbf{q}} \sum_{\mathbf{p}} \int_{-\infty}^{t} \mathrm{~d} t^{\prime} \exp \left\{\epsilon\left(t^{\prime}-t\right)\right\} T_{\mathbf{q}}\left(t, t^{\prime}\right) \\
& \times \int_{0}^{1} \mathrm{~d} \tau\left(\rho_{\mathrm{q}}\left(t^{\prime}\right)\right)^{\tau} I_{n}\left(\mathbf{p}, \mathbf{q}, t^{\prime}\right)\left(\rho_{\mathrm{q}}\left(t^{\prime}\right)\right)^{1-\tau} \gamma_{-\mathbf{q}}\left(\mathbf{p}, t^{\prime}\right) .
\end{aligned}
$$

The nonequilibrium statistical operator (2.30) is obtained at a consistent description of kinetics and hydrodynamics of the Bose system. Using it we shall find the non-closed system of transport equations for the parameters of an abbreviated description $f_{1}(\mathbf{p}, \mathbf{q}, t),\left\langle\hat{\varepsilon}_{\mathbf{q}}^{\text {int }}\right\rangle t$. For this purpose we use the identities:

$$
\begin{aligned}
\frac{\partial}{\partial t} f_{1}(\mathbf{p}, \mathbf{q}, t) & =\frac{\partial}{\partial t}\left\langle\hat{n}_{\mathbf{q}}(\mathbf{p})\right\rangle^{t}=\left\langle\dot{\hat{n}}_{\mathbf{q}}(\mathbf{p})\right\rangle_{\mathbf{q}}^{t}+\left\langle I_{n}(\mathbf{p}, \mathbf{q})\right\rangle^{t} \\
\frac{\partial}{\partial t}\left\langle\hat{\varepsilon}_{\mathbf{q}}^{\text {int }}\right\rangle^{t} & =\left\langle\dot{\hat{\varepsilon}}_{\mathbf{q}}^{\text {int }}\right\rangle^{t}=\left\langle\dot{\hat{\varepsilon}}_{\mathbf{q}}^{\text {int }}\right\rangle_{\mathbf{q}}^{t}+\left\langle I_{\varepsilon}^{\text {int }}(\mathbf{q})\right\rangle^{t}
\end{aligned}
$$

where

$$
\dot{\hat{n}}_{\mathbf{q}}(\mathbf{p})=\mathrm{i} \hat{L}_{N} \hat{n}_{\mathbf{q}}(\mathbf{p}), \quad \dot{\hat{\varepsilon}}_{\mathbf{q}}^{\text {int }}=\mathrm{i} \hat{L}_{N} \hat{\varepsilon}_{\mathbf{q}}^{\text {int }} .
$$

Now we shall perform the averaging in right parts (2.31) with the nonequilibrium statistical operator (2.30). As a result, we shall find the set of equations for 
the nonequilibrium distribution function $f_{1}(\mathbf{p}, \mathbf{q}, t)$ and average value of interaction energy density $\left\langle\hat{\varepsilon}_{\mathbf{q}}^{\text {int }}\right\rangle^{t}$ :

$$
\begin{aligned}
\frac{\partial}{\partial t}\left\langle\hat{n}_{\mathbf{q}}(\mathbf{p})\right\rangle^{t}= & \left\langle\dot{\hat{n}}_{\mathbf{q}}(\mathbf{p})\right\rangle_{\mathbf{q}}^{t}+\sum_{\mathbf{q}^{\prime}} \int_{-\infty}^{t} \mathrm{~d} t^{\prime} \exp \left\{\epsilon\left(t^{\prime}-t\right)\right\} \varphi_{n \varepsilon}^{\text {int }}\left(\mathbf{q}, \mathbf{p}, \mathbf{q}^{\prime}, t, t^{\prime}\right) \beta_{-\mathbf{q}}\left(t^{\prime}\right) \\
& +\sum_{\mathbf{q}^{\prime}} \sum_{\mathbf{p}^{\prime}} \int_{-\infty}^{t} \mathrm{~d} t^{\prime} \exp \left\{\epsilon\left(t^{\prime}-t\right)\right\} \varphi_{n n}\left(\mathbf{q}, \mathbf{p}, \mathbf{q}^{\prime}, \mathbf{q}^{\prime}, t, t^{\prime}\right) \gamma_{-\mathbf{q}}\left(\mathbf{p}^{\prime}, t^{\prime}\right) \\
\frac{\partial}{\partial t}\left\langle\hat{\varepsilon}_{\mathbf{q}}^{\text {int }}\right\rangle^{t}= & \left\langle\dot{\hat{\varepsilon}}_{\mathbf{q}}^{\text {int }}\right\rangle_{\mathbf{q}}^{t}+\sum_{\mathbf{q}^{\prime}} \int_{-\infty}^{t} \mathrm{~d} t^{\prime} \exp \left\{\epsilon\left(t^{\prime}-t\right)\right\} \varphi_{\varepsilon \varepsilon}^{\text {int }}\left(\mathbf{q}, \mathbf{q}^{\prime}, t, t^{\prime}\right) \beta_{-\mathbf{q}}\left(t^{\prime}\right) \\
& +\sum_{\mathbf{q}^{\prime}} \sum_{\mathbf{p}^{\prime}} \int_{-\infty}^{t} \mathrm{~d} t^{\prime} \exp \left\{\epsilon\left(t^{\prime}-t\right)\right\} \varphi_{\varepsilon n}^{\text {int }}\left(\mathbf{q}, \mathbf{q}^{\prime}, \mathbf{p}^{\prime}, t, t^{\prime}\right) \gamma_{-\mathbf{q}}\left(\mathbf{p}^{\prime}, t^{\prime}\right)
\end{aligned}
$$

In the equations (2.33) the generalized transport kernels are introduced, which describe dissipative processes in the system:

$$
\begin{aligned}
\varphi_{n \varepsilon}^{\text {int }}\left(\mathbf{q}, \mathbf{p}, \mathbf{q}^{\prime}, t, t^{\prime}\right) & =\operatorname{Sp}\left[I_{n}(\mathbf{q}, \mathbf{p}, t) T_{\mathrm{q}}\left(t, t^{\prime}\right) \int_{0}^{1} \mathrm{~d} \tau \rho_{\mathrm{q}}^{\tau}\left(t^{\prime}\right) I_{\varepsilon}^{\mathrm{int}}\left(\mathbf{q}^{\prime}, t^{\prime}\right) \rho_{\mathrm{q}}^{1-\tau}\left(t^{\prime}\right)\right], \\
\varphi_{\varepsilon n}^{\text {int }}\left(\mathbf{q}, \mathbf{q}^{\prime}, \mathbf{p}, t, t^{\prime}\right) & =\operatorname{Sp}\left[I_{\varepsilon}^{\text {int }}\left(\mathbf{q}, t^{\prime}\right) T_{\mathbf{q}}\left(t, t^{\prime}\right) \int_{0}^{1} \mathrm{~d} \tau \rho_{\mathrm{q}}^{\tau}\left(t^{\prime}\right) I_{n}\left(\mathbf{p}, \mathbf{q}^{\prime}, t\right) \rho_{\mathrm{q}}^{1-\tau}\left(t^{\prime}\right)\right], \\
\varphi_{n n}\left(\mathbf{q}, \mathbf{p}, \mathbf{q}^{\prime}, \mathbf{p}^{\prime}, t, t^{\prime}\right) & =\operatorname{Sp}\left[I_{n}(\mathbf{q}, \mathbf{p}, t) T_{\mathrm{q}}\left(t, t^{\prime}\right) \int_{0}^{1} \mathrm{~d} \tau \rho_{\mathrm{q}}^{\tau}\left(t^{\prime}\right) I_{n}\left(\mathbf{p}^{\prime}, \mathbf{q}^{\prime}, t\right) \rho_{\mathrm{q}}^{1-\tau}\left(t^{\prime}\right)\right], \\
\varphi_{\varepsilon \varepsilon}^{\text {int }}\left(\mathbf{q}, \mathbf{q}^{\prime}, t, t^{\prime}\right) & =\operatorname{Sp}\left[I_{\varepsilon}^{\text {int }}\left(\mathbf{q}, t^{\prime}\right) T_{\mathrm{q}}\left(t, t^{\prime}\right) \int_{0}^{1} \mathrm{~d} \tau \rho_{\mathrm{q}}^{\tau}\left(t^{\prime}\right) I_{\varepsilon}^{\text {int }}\left(\mathbf{q}^{\prime}, t^{\prime}\right) \rho_{\mathrm{q}}^{1-\tau}\left(t^{\prime}\right)\right] .
\end{aligned}
$$

The system of equations (2.33) for the one-particle distribution function and average density of potential energy presents a strongly nonlinear system and can be used for the description of both strongly and weakly nonequilibrium states of the Bose system at a consistent description of kinetics and hydrodynamics. These transport equations for the Bose systems are new. Projecting them on the values of components of the vector $\Psi(\mathbf{p})=(1, \mathbf{p}, p 2 / 2 m-q 2 / 8 m)$, according to (2.14)-(2.16), we shall receive the equations of nonlinear hydrodynamics in which the transport processes of kinetic and potential parts of energy are described by two interdependent equations.

It is obvious that such equations of hydrodynamics of nonlinear processes enable us to describe more in detail the processes of mutual transformation of kinetic and potential energy of particles at the transition from a vapour to a liquid state at the change of density, pressure and temperatures.

The system of the transport equations becomes considerably simpler and is closed for weakly nonequilibrium processes. We shall consider such a case in the next section. 


\section{Kinetics and hydrodynamics of nonequilibrium state near equilibrium}

For kinetics and hydrodynamics of nonequilibrium processes which are close to an equilibrium state, the parameters of an abbreviated description $\left\langle\hat{n}_{\mathbf{q}}(\mathbf{p})\right\rangle^{t},\left\langle\hat{\varepsilon}_{\mathbf{q}}^{\text {int }}\right\rangle^{t}$ slowly change in space and time and slightly differ from their equilibrium values. Then it is sufficient to consider the deviations of parameters $\beta_{\mathbf{q}}(t), \gamma_{\mathbf{q}}(\mathbf{p}, t)$ from equilibrium values using the linear approximation. In this approximation, the generalized transport equations (2.33) [39] transform into a transport equation for $f_{\mathbf{k}}(\mathbf{p} ; t)=\left\langle\hat{n}_{\mathbf{k}}(\mathbf{p})\right\rangle^{t}$, $h_{\mathbf{k}}^{\text {int }}(t)=\left\langle\hat{h}_{\mathbf{k}}^{\text {int }}\right\rangle^{t}$

$$
\begin{aligned}
& \frac{\partial}{\partial t} f_{\mathbf{k}}(\mathbf{p} ; t)+\frac{\mathrm{ik} \cdot \mathbf{p}}{m} f_{\mathbf{k}}(\mathbf{p} ; t)= \\
& =-\frac{\mathrm{i} \mathbf{k} \cdot \mathbf{p}}{m} n f_{0}(p) c_{2}(\mathbf{k}) \sum_{\mathbf{p}^{\prime}} f_{\mathbf{k}}\left(\mathbf{p}^{\prime} ; t\right)+\mathrm{i} \Omega_{n h}(\mathbf{k}, \mathbf{p}) h_{\mathbf{k}}^{\mathrm{int}}(t) \\
& \quad-\sum_{\mathbf{p}^{\prime}} \int_{-\infty}^{t} \mathrm{~d} t^{\prime} \mathrm{e}^{\varepsilon\left(t^{\prime}-t\right)} \varphi_{n n}\left(\mathbf{k}, \mathbf{p}, \mathbf{p}^{\prime} ; t, t^{\prime}\right) f_{\mathbf{k}}\left(\mathbf{p}^{\prime} ; t^{\prime}\right) \\
& \quad-\int_{-\infty}^{t} \mathrm{~d} t^{\prime} \mathrm{e}^{\varepsilon\left(t^{\prime}-t\right)} \varphi_{n h}\left(\mathbf{k}, \mathbf{p} ; t, t^{\prime}\right) h_{\mathbf{k}}^{\text {int }}\left(t^{\prime}\right) \\
& \frac{\partial}{\partial t} h_{\mathbf{k}}^{\text {int }}(t)=\sum_{\mathbf{p}} \mathrm{i}^{\mathrm{i}} \Omega_{h n}(\mathbf{k}, \mathbf{p}) f_{\mathbf{k}}(\mathbf{p} ; t)-\sum_{\mathbf{p}} \int_{-\infty}^{t} \mathrm{~d} t^{\prime} \mathrm{e}^{\varepsilon\left(t^{\prime}-t\right)} \varphi_{h n}\left(\mathbf{k}, \mathbf{p} ; t, t^{\prime}\right) f_{\mathbf{k}}\left(\mathbf{p} ; t^{\prime}\right) \\
& -\int_{-\infty}^{t} \mathrm{~d} t^{\prime} \mathrm{e}^{\varepsilon\left(t^{\prime}-t\right)} \varphi_{h h}\left(\mathbf{k} ; t, t^{\prime}\right) h_{\mathbf{k}}^{\text {int }}\left(t^{\prime}\right)
\end{aligned}
$$

where $\mathrm{i} \Omega_{n h}(\mathbf{k}, \mathbf{p}), \mathrm{i} \Omega_{h n}(\mathbf{k}, \mathbf{p})$ are normalized static correlation functions:

$$
\begin{aligned}
\mathrm{i} \Omega_{n h}(\mathbf{k}, \mathbf{p}) & =\left\langle\dot{\hat{n}}_{\mathbf{k}}(\mathbf{p}) \hat{h}_{-\mathbf{k}}^{\mathrm{int}}\right\rangle_{0} \Phi_{h h}^{-1}(\mathbf{k}), \\
\mathrm{i} \Omega_{h n}(\mathbf{k}, \mathbf{p}) & =\sum_{\mathbf{p}^{\prime}}\left\langle\dot{\hat{h}}_{\mathbf{k}} \hat{n}_{-\mathbf{k}}\left(\mathbf{p}^{\prime}\right)\right\rangle_{0} \Phi_{\mathbf{k}}^{-1}\left(\mathbf{p}^{\prime}, \mathbf{p}\right),
\end{aligned}
$$

where

$$
\begin{aligned}
& \hat{h}_{\mathbf{k}}^{\text {int }}=\hat{\varepsilon}_{\mathbf{k}}-\int \mathrm{d} \mathbf{p d} \mathbf{p}^{\prime}\left\langle\hat{\varepsilon}_{\mathbf{k}} \hat{n}_{-\mathbf{k}}\left(\mathbf{p}^{\prime}\right)\right\rangle_{0} \Phi_{\mathbf{k}}^{-1}\left(\mathbf{p}^{\prime}, \mathbf{p}\right) \hat{n}_{\mathbf{k}}(\mathbf{p})=\hat{\varepsilon}_{\mathbf{k}}^{\text {int }}-\left\langle\hat{\varepsilon}_{\mathbf{k}}^{\text {int }} \hat{n}_{-\mathbf{k}}\right\rangle_{0} S_{2}^{-1}(\mathbf{k}) \hat{n}_{\mathbf{k}}, \\
& \hat{\varepsilon}_{\mathbf{k}}^{\text {int }}=\frac{1}{2} \sum_{l \neq j=1}^{N} \Phi\left(\left|\mathbf{r}_{l j}\right|\right) \mathrm{e}^{-\mathrm{ik} \cdot \mathbf{r}_{l}}, \quad \hat{n}_{\mathbf{k}}=\sum_{l=1}^{N} \mathrm{e}^{-\mathrm{i} \mathbf{k} \cdot \mathbf{r}_{l}}
\end{aligned}
$$

are the Fourier-components of densities for the interaction energy and the number of particles, respectively. Further, it is more convenient to use instead of the dynamical variable of energy $\hat{\varepsilon}_{\mathbf{k}}$ the variable $\hat{h}_{\mathbf{k}}^{\text {int }}(3.5)$ which is orthogonal to $\hat{n}_{\mathbf{k}}(\mathbf{p})$ by means of the equality:

$$
\left\langle\hat{h}_{\mathbf{k}}^{\mathrm{int}} \hat{n}_{-\mathbf{k}}\right\rangle_{0}=0
$$


From the structure of the dynamical variable $\hat{h}_{\mathbf{k}}^{\text {int }}(3.3)$ it can be seen that it corresponds to a potential part of the Fourier-component of the generalized enthalpy $\hat{h}_{\mathbf{k}}$ which is introduced in the molecular hydrodynamics $[25,26]$ :

$$
\hat{h}_{\mathbf{k}}=\hat{\varepsilon}_{\mathbf{k}}-\left\langle\hat{\varepsilon}_{\mathbf{k}} \hat{n}_{-\mathbf{k}}\right\rangle_{0} \hat{n}_{\mathbf{k}}=\hat{h}_{\mathbf{k}}^{\mathrm{kin}}+\hat{h}_{\mathbf{k}}^{\mathrm{int}},
$$

where

$$
\hat{h}_{\mathbf{k}}^{\text {kin }}=\hat{\varepsilon}_{\mathbf{k}}^{\text {kin }}-\left\langle\hat{\varepsilon}_{\mathbf{k}}^{\text {kin }} \hat{n}_{-\mathbf{k}}\right\rangle_{0} S_{2}^{-1}(\mathbf{k}) \hat{n}_{\mathbf{k}}
$$

is a kinetic part of the generalized enthalpy,

$$
\hat{\varepsilon}_{\mathbf{k}}^{\mathrm{kin}}=\sum_{l=1}^{N} \frac{p_{l}^{2}}{2 m} \mathrm{e}^{-\mathbf{k} \cdot \mathbf{r}_{l}}
$$

is the Fourier-component of the kinetic energy density. $S_{2}=\left\langle\hat{n}_{\mathbf{k}} \hat{n}_{-\mathbf{k}}\right\rangle_{0}$ is the static structure factor of the system.

$$
\Phi_{\mathbf{k}}^{-1}\left(\mathbf{p}^{\prime \prime}, \mathbf{p}^{\prime}\right)=\frac{\delta\left(\mathbf{p}^{\prime \prime}-\mathbf{p}^{\prime}\right)}{n f_{0}\left(p^{\prime \prime}\right)}-c_{2}(\mathbf{k}),
$$

where $c_{2}(\mathbf{k})$ denotes a direct correlation function which is connected with the correlation function $h_{2}(\mathbf{k})$ as: $h_{2}(\mathbf{k})=c_{2}(\mathbf{k})\left[1-n c_{2}(\mathbf{k})\right]^{-1}$.

$$
\begin{aligned}
\varphi_{n n}\left(\mathbf{k}, \mathbf{p}, \mathbf{p}^{\prime} ; t, t^{\prime}\right) & =\sum_{\mathbf{p}^{\prime \prime}}\left\langle I_{n}(\mathbf{k}, \mathbf{p}) T_{0}\left(t, t^{\prime}\right) I_{n}\left(-\mathbf{k}, \mathbf{p}^{\prime \prime}\right)\right\rangle_{0} \Phi_{\mathbf{k}}^{-1}\left(\mathbf{p}^{\prime \prime}, \mathbf{p}^{\prime}\right), \\
\varphi_{h n}\left(\mathbf{k}, \mathbf{p} ; t, t^{\prime}\right) & =\sum_{\mathbf{p}^{\prime}}\left\langle I_{h}^{\mathrm{int}}(\mathbf{k}) T_{0}\left(t, t^{\prime}\right) I_{n}\left(-\mathbf{k}, \mathbf{p}^{\prime}\right)\right\rangle_{0} \Phi_{\mathbf{k}}^{-1}\left(\mathbf{p}^{\prime}, \mathbf{p}\right), \\
\varphi_{n h}\left(\mathbf{k}, \mathbf{p} ; t, t^{\prime}\right) & =\left\langle I_{n}(\mathbf{k}, \mathbf{p}) T_{0}\left(t, t^{\prime}\right) I_{h}^{\mathrm{int}}(-\mathbf{k})\right\rangle_{0} \Phi_{h h}^{-1}(\mathbf{k}), \\
\varphi_{h h}\left(\mathbf{k} ; t, t^{\prime}\right) & =\left\langle I_{h}^{\mathrm{int}}(\mathbf{k}) T_{0}\left(t, t^{\prime}\right) I_{h}^{\mathrm{int}}(-\mathbf{k})\right\rangle_{0} \Phi_{h h}^{-1}(\mathbf{k})
\end{aligned}
$$

are the generalized transport kernels (memory functions) which describe kinetic and hydrodynamic processes.

$$
\begin{aligned}
I_{n}(\mathbf{k}, \mathbf{p}) & =\left(1-\mathcal{P}_{0}\right) \dot{\hat{n}}_{\mathbf{k}}(\mathbf{p}), \\
I_{h}^{\mathrm{int}}(\mathbf{k}) & =\left(1-\mathcal{P}_{0}\right) \hat{\hat{h}}_{\mathbf{k}}^{\mathrm{int}}
\end{aligned}
$$

are the generalized fluxes in the linear approximation, $\dot{\hat{n}}_{\mathbf{k}}(\mathbf{p})=\mathrm{i} L_{N} \hat{n}_{\mathbf{k}}(\mathbf{p}), \dot{\hat{h}}_{\mathbf{k}}^{\text {int }}=$ $\mathrm{i} L_{N} \hat{h}_{\mathbf{k}}^{\text {int }}, T_{0}\left(t, t^{\prime}\right)=\mathrm{e}^{\left(t-t^{\prime}\right)\left(1-\mathcal{P}_{0}\right) i L_{N}}$ is the time evolution operator with the projection operator $\mathcal{P}_{0}$ which acts on the dynamical variables $\hat{A}_{\mathbf{k}}$

$$
\mathcal{P}_{0} \hat{A}_{\mathbf{k}^{\prime}}=\sum_{\mathbf{k}^{\prime}}\left\langle\hat{A}_{\mathbf{k}^{\prime}} \hat{h}_{-\mathbf{k}}^{\mathrm{int}}\right\rangle_{0} \Phi_{h h}^{-1}(\mathbf{k}) \hat{h}_{\mathbf{k}}^{\mathrm{int}}+\sum_{\mathbf{k}} \sum_{\mathbf{p p}^{\prime}}\left\langle\hat{A}_{\mathbf{k}^{\prime}} \hat{n}_{-\mathbf{k}}\left(\mathbf{p}^{\prime}\right)\right\rangle_{0} \Phi_{\mathbf{k}}^{-1}\left(\mathbf{p}^{\prime}, \mathbf{p}\right) \hat{n}_{\mathbf{k}}(\mathbf{p})
$$

The system of transport equations (3.1), (3.2) is closed. We shall use the Laplace transform with respect to time, assuming that at $t>0$ the quantities $f_{\mathbf{k}}(\mathbf{p} ; t=0)$, $h_{\mathbf{k}}^{\text {int }}(t=0)$ are known

$$
A(z)=\mathrm{i} \int_{0}^{\infty} \mathrm{d} t \mathrm{e}^{\mathrm{i} z t} A(t), \quad z=\omega+\mathrm{i} \varepsilon, \quad \varepsilon \rightarrow+0 .
$$


Then, equations (3.1) and (3.2) are presented in the form:

$$
\begin{aligned}
& z f_{\mathbf{k}}(\mathbf{p} ; z)+\frac{\mathrm{i} \mathbf{k} \cdot \mathbf{p}}{m} f_{\mathbf{k}}(\mathbf{p} ; z)=-\frac{\mathrm{i} \mathbf{k} \cdot \mathbf{p}}{m} n f_{0}(p) c_{2}(\mathbf{k}) \\
& \times \sum_{\mathbf{p}^{\prime}} f_{\mathbf{k}}\left(\mathbf{p}^{\prime} ; z\right)+\Sigma_{n h}(\mathbf{k}, \mathbf{p} ; z) h_{\mathbf{k}}^{\mathrm{int}}(z) \\
&-\sum_{\mathbf{p}^{\prime}} \varphi_{n n}\left(\mathbf{k}, \mathbf{p}, \mathbf{p}^{\prime} ; z\right) f_{\mathbf{k}}\left(\mathbf{p}^{\prime} ; z\right)+f_{\mathbf{k}}(\mathbf{p} ; t=0) \\
& z h_{\mathbf{k}}^{\text {int }}(z)=\sum_{\mathbf{p}^{\prime}} \Sigma_{h n}\left(\mathbf{k}, \mathbf{p}^{\prime} ; z\right) f_{\mathbf{k}}\left(\mathbf{p}^{\prime} ; z\right)-\varphi_{h h}(\mathbf{k} ; z) h_{\mathbf{k}}^{\text {int }}(z)+h_{\mathbf{k}}^{\text {int }}(t=0) \\
& \Sigma_{n h}(\mathbf{k}, \mathbf{p} ; z)= \\
& \Sigma_{h n}(\mathbf{k}, \mathbf{p} ; z)= \mathrm{i} \Omega_{n h}(\mathbf{k}, \mathbf{p})-\varphi_{h n}(\mathbf{k}, \mathbf{p})-\varphi_{h n}(\mathbf{k}, \mathbf{p} ; z)
\end{aligned}
$$

In the next subsection, based on the system of transport equations for Fourier components of the nonequilibrium one-particle distribution function and the potential part of enthalpy (3.21), (3.22), we shall obtain equations for time correlation functions. We shall also investigate the spectrum of collective excitations and the structure of generalized transport coefficients.

\section{Time correlation functions, collective modes and general- ized transport coefficients}

Using combined equations (3.1), (3.2) one obtains a system for time correlation functions:

$$
\begin{aligned}
\Phi_{n n}\left(\mathbf{k}, \mathbf{p}, \mathbf{p}^{\prime} ; t\right) & =\sum_{\mathbf{p}^{\prime \prime}}\left\langle\hat{n}_{\mathbf{k}}(\mathbf{p} ; t) \hat{n}_{-\mathbf{k}}\left(\mathbf{p}^{\prime \prime} ; 0\right)\right\rangle_{0} \Phi_{\mathbf{k}}^{-1}\left(\mathbf{p}^{\prime \prime}, \mathbf{p}^{\prime}\right) \\
\Phi_{h n}(\mathbf{k}, \mathbf{p} ; t) & =\sum_{\mathbf{p}^{\prime}}\left\langle\hat{h}_{\mathbf{k}}^{\mathrm{int}}(t) \hat{n}_{-\mathbf{k}}\left(\mathbf{p}^{\prime} ; 0\right)\right\rangle_{0} \Phi_{\mathbf{k}}^{-1}\left(\mathbf{p}^{\prime}, \mathbf{p}\right) \\
\Phi_{n h}(\mathbf{k}, \mathbf{p} ; t) & =\left\langle\hat{n}_{\mathbf{k}}(\mathbf{p} ; t) \hat{h}_{-\mathbf{k}}^{\mathrm{int}}(0)\right\rangle_{0} \Phi_{h h}^{-1}(\mathbf{k}) \\
\Phi_{h h}(\mathbf{k} ; t) & =\left\langle\hat{h}_{\mathbf{k}}^{\mathrm{int}}(t) \hat{h}_{-\mathbf{k}}^{\mathrm{int}}(0)\right\rangle_{0} \Phi_{h h}^{-1}(\mathbf{k})
\end{aligned}
$$

where $\hat{n}_{\mathbf{k}}(\mathbf{p} ; t)=\mathrm{e}^{-\mathrm{i} L_{N} t} \hat{n}_{\mathbf{k}}(\mathbf{p} ; 0), \hat{h}_{\mathbf{k}}^{\mathrm{int}}(t)=\mathrm{e}^{-\mathrm{i} L_{N} t} \hat{h}_{\mathbf{k}}^{\mathrm{int}}(0)$.

One uses the Fourier transform with respect to time

$$
\langle a\rangle_{\omega}=\int_{-\infty}^{\infty} \mathrm{d} t \mathrm{e}^{\mathrm{i} \omega t}\langle a\rangle^{t} .
$$

Then we write the system of equations (4.1), (4.2) in the form:

$$
\begin{aligned}
-\mathrm{i} \omega\left\langle\hat{n}_{\mathbf{k}}(\mathbf{p})\right\rangle_{\omega} & =\sum_{\mathbf{p}^{\prime}} \Sigma_{n n}\left(\mathbf{k}, \mathbf{p}, \mathbf{p}^{\prime} ; \omega+\mathrm{i} \varepsilon\right)\left\langle\hat{n}_{\mathbf{k}}\left(\mathbf{p}^{\prime}\right)\right\rangle_{\omega}+\Sigma_{n h}(\mathbf{k}, \mathbf{p} ; \omega+\mathrm{i} \varepsilon)\left\langle\hat{h}_{\mathbf{k}}^{\mathrm{int}}\right\rangle_{\omega}, \\
-\mathrm{i} \omega\left\langle\hat{h}_{\mathbf{k}}^{\mathrm{int}}\right\rangle_{\omega} & =\sum_{\mathbf{p}^{\prime}} \Sigma_{h n}\left(\mathbf{k}, \mathbf{p}^{\prime} ; \omega+\mathrm{i} \varepsilon\right)\left\langle\hat{n}_{\mathbf{k}}\left(\mathbf{p}^{\prime}\right)\right\rangle_{\omega}-\varphi_{h h}(\mathbf{k} ; \omega+\mathrm{i} \varepsilon)\left\langle\hat{h}_{\mathbf{k}}^{\mathrm{int}}\right\rangle_{\omega},
\end{aligned}
$$


where

$$
\begin{aligned}
\Sigma_{n n}\left(\mathbf{k}, \mathbf{p}, \mathbf{p}^{\prime} ; \omega+\mathrm{i} \varepsilon\right) & =\mathrm{i} \Omega_{n n}\left(\mathbf{k}, \mathbf{p}, \mathbf{p}^{\prime}\right)-\varphi_{n n}\left(\mathbf{k}, \mathbf{p}, \mathbf{p}^{\prime} ; \omega+\mathrm{i} \varepsilon\right), \\
\Sigma_{n h}(\mathbf{k}, \mathbf{p} ; \omega+\mathrm{i} \varepsilon) & =\mathrm{i} \Omega_{n h}(\mathbf{k}, \mathbf{p})-\varphi_{n h}(\mathbf{k}, \mathbf{p} ; \omega+\mathrm{i} \varepsilon) \\
\Sigma_{h n}(\mathbf{k}, \mathbf{p} ; \omega+\mathrm{i} \varepsilon) & =\mathrm{i} \Omega_{h n}(\mathbf{k}, \mathbf{p})-\varphi_{h n}(\mathbf{k}, \mathbf{p} ; \omega+\mathrm{i} \varepsilon)
\end{aligned}
$$

It is more convenient to present the system of equations (4.5), (4.6) in a matrix form:

$$
-\mathrm{i} \omega\left\langle\tilde{a}_{\mathbf{k}}\right\rangle_{\omega}=\tilde{\Sigma}(\mathbf{k} ; \omega+\mathrm{i} \varepsilon)\left\langle\tilde{a}_{\mathbf{k}}\right\rangle_{\omega},
$$

where $\tilde{a}_{\mathbf{k}}=\operatorname{col}\left(\hat{n}_{\mathbf{k}}(\mathbf{p}), \hat{h}_{\mathbf{k}}^{\text {int }}\right)$ is a vector-column and

$$
\begin{aligned}
& \tilde{\Sigma}(\mathbf{k} ; \omega+\mathrm{i} \varepsilon)=\left[\begin{array}{lll}
\sum_{\mathbf{p}^{\prime}} & \Sigma_{n n}\left(\mathbf{k}, \mathbf{p}, \mathbf{p}^{\prime} ; \omega+\mathrm{i} \varepsilon\right) & \Sigma_{n h}(\mathbf{k}, \mathbf{p} ; \omega+\mathrm{i} \varepsilon) \\
\sum_{\mathbf{p}^{\prime}} & \Sigma_{h n}\left(\mathbf{k}, \mathbf{p}^{\prime} ; \omega+\mathrm{i} \varepsilon\right) & -\varphi_{h h}(\mathbf{k} ; \omega+\mathrm{i} \varepsilon)
\end{array}\right], \\
& \tilde{\Sigma}(\mathbf{k} ; \omega+\mathrm{i} \varepsilon)=\int_{0}^{\infty} \mathrm{d} t \mathrm{e}^{\mathrm{i}(\omega+\mathrm{i} \varepsilon) t} \tilde{\Sigma}(\mathbf{k} ; t) .
\end{aligned}
$$

Now, one uses the solution to the Liouville equation in approximation [40,41] without introducing the projection operator $\mathcal{P}_{\mathrm{q}}(t)$ :

$$
\varrho\left(x^{N} ; t\right)=\varrho_{\mathrm{q}}\left(x^{N} ; t\right)-\int_{-\infty}^{t} \mathrm{~d} t^{\prime} \mathrm{e}^{\varepsilon\left(t^{\prime}-t\right)} \mathrm{e}^{\mathrm{i} L_{N}\left(t^{\prime}-t\right)}\left(\frac{\partial^{\prime}}{\partial t}+\mathrm{i} L_{N}\right) \varrho_{\mathrm{q}}\left(x^{N} ; t^{\prime}\right) .
$$

Then, from the self-consistency conditions $\left\langle\tilde{a}_{\mathbf{k}}\right\rangle^{t}=\left\langle\tilde{a}_{\mathbf{k}}\right\rangle_{\mathrm{q}}^{t}$ we obtain a system of equations which connects the average values $\left\langle\hat{n}_{\mathbf{k}}(\mathbf{p})\right\rangle_{\omega}$ and $\left\langle\hat{h}_{\mathbf{k}}^{\mathrm{int}}\right\rangle_{\omega}$ with spectral functions of time correlation functions:

$$
\mathrm{i} \omega \tilde{\Phi}(\mathbf{k} ; \omega+\mathrm{i} \varepsilon)\left\langle\tilde{a}_{\mathbf{k}}\right\rangle_{\omega}=[\tilde{\Phi}(\mathbf{k})-\mathrm{i}(\omega+\mathrm{i} \varepsilon) \tilde{\Phi}(\mathbf{k} ; \omega+\mathrm{i} \varepsilon)]\left\langle\tilde{a}_{\mathbf{k}}\right\rangle_{\omega}
$$

where

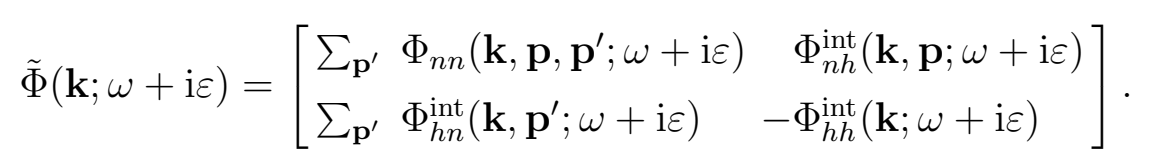

Let us multiply equation (4.10) by the matrix $\tilde{\Phi}(\mathbf{k} ; \omega+\mathrm{i} \varepsilon)$ and compare the result with equation (4.12). So we find

$$
\begin{aligned}
z \tilde{\Phi}(\mathbf{k} ; z) & =\tilde{\Sigma}(\mathbf{k} ; z) \tilde{\Phi}(\mathbf{k} ; z)-\tilde{\Phi}(\mathbf{k}), & & z=\omega+\mathrm{i} \varepsilon \\
\tilde{\Phi}(\mathbf{k} ; z) & =\mathrm{i} \int_{0}^{\infty} \mathrm{d} t \mathrm{e}^{\mathrm{i} z t} \tilde{\Phi}(\mathbf{k} ; t), & & \varepsilon \rightarrow 0
\end{aligned}
$$

or in an explicit form:

$$
\begin{aligned}
z \Phi_{n n}\left(\mathbf{k}, \mathbf{p}, \mathbf{p}^{\prime} ; z\right)= & \sum_{\mathbf{p}^{\prime \prime}} \Sigma_{n n}\left(\mathbf{k}, \mathbf{p}, \mathbf{p}^{\prime \prime} ; z\right) \Phi_{n n}\left(\mathbf{k}, \mathbf{p}^{\prime \prime}, \mathbf{p}^{\prime} ; z\right) \\
& +\Sigma_{n h}(\mathbf{k}, \mathbf{p} ; z) \Phi_{h n}^{\mathrm{int}}\left(\mathbf{k}, \mathbf{p}^{\prime} ; z\right)-\Phi_{n n}\left(\mathbf{k}, \mathbf{p}, \mathbf{p}^{\prime}\right)
\end{aligned}
$$




$$
\begin{aligned}
z \Phi_{n h}^{\mathrm{int}}(\mathbf{k}, \mathbf{p} ; z)= & \sum_{\mathbf{p}^{\prime \prime}} \Sigma_{n n}\left(\mathbf{k}, \mathbf{p}, \mathbf{p}^{\prime \prime} ; z\right) \Phi_{n h}^{\mathrm{int}}\left(\mathbf{k}, \mathbf{p}^{\prime \prime} ; z\right) \\
& +\Sigma_{n h}(\mathbf{k}, \mathbf{p} ; z) \Phi_{h h}^{\mathrm{int}}(\mathbf{k} ; z), \\
z \Phi_{h n}^{\mathrm{int}}\left(\mathbf{k}, \mathbf{p}^{\prime} ; z\right)= & \sum_{\mathbf{p}^{\prime \prime}} \Sigma_{h n}\left(\mathbf{k}, \mathbf{p}^{\prime \prime} ; z\right) \Phi_{n n}^{\mathrm{int}}\left(\mathbf{k}, \mathbf{p}^{\prime \prime}, \mathbf{p}^{\prime} ; z\right) \\
& -\varphi_{h h}(\mathbf{k} ; z) \Phi_{h n}^{\mathrm{int}}\left(\mathbf{k}, \mathbf{p}^{\prime} ; z\right), \\
z \Phi_{h h}^{\mathrm{int}}(\mathbf{k} ; z)= & \sum_{\mathbf{p}^{\prime \prime}} \Sigma_{h n}\left(\mathbf{k}, \mathbf{p}^{\prime \prime} ; z\right) \Phi_{n h}^{\mathrm{int}}\left(\mathbf{k}, \mathbf{p}^{\prime \prime} ; z\right) \\
& -\varphi_{h h}(\mathbf{k} ; z) \Phi_{h h}^{\mathrm{int}}(\mathbf{k} ; z)-\Phi_{h h}(\mathbf{k}),
\end{aligned}
$$

where the condition $\Phi_{h n}\left(\mathbf{k}, \mathbf{p}^{\prime}\right)=\Phi_{n h}(\mathbf{k}, \mathbf{p})=0$ is taken into account.

The system of equations (4.14)-(4.17) for time correlation functions of weakly nonequilibrium Bose system is obtained based on a consistent description of kinetics and hydrodynamics. These are new equations for the quantum Bose-system. Projecting them on eigenvalues $(1, \mathbf{p})$ of quantum one-partial distribution function $f_{1}(\mathbf{p}, \mathbf{q}, t)$ we shall obtain the corresponding system of equations for time correlation functions which can be derived based on the equations of molecular hydrodynamics or the method of Green functions [10] (in view of the connection of time correlation functions with corresponding Green time functions).

Moreover, designing the system of equations (4.14)-(4.17) for higher moments of the quantum one-partial distribution function results in the corresponding systems of hydrodynamical equations for the time correlation functions related to the densities of operators of particle number, momentum, enthalpy, generalized tensor of viscous tension and generalized flow of enthalpy. Similar equations for the Green time functions have been obtained in papers by Tserkovnikov [10,13,15]. A principal interest in such an approach is the study of collective modes and generalized transport coefficients such as viscosity and thermal conductivity of quantum Bose systems.

In order to solve the system of equations (4.14)-(4.17) we also apply the projection procedure $[42,43]$. Let us introduce the dimensionless momentum $\xi=\mathbf{k}\left(m v_{0}\right)^{-1}$, $v_{0}^{2}=(m \beta)^{-1}$. Then the system of equations (4.13)-(4.17) can be rewritten in the matrix form [43]:

$$
z \tilde{\Phi}\left(\mathbf{k} ; \xi, \xi^{\prime} ; z\right)-\tilde{\Sigma}\left(\mathbf{k} ; \xi, \xi^{\prime \prime} ; z\right) \tilde{\Phi}\left(\mathbf{k} ; \xi^{\prime \prime}, \xi^{\prime} ; z\right)=-\tilde{\Phi}\left(\mathbf{k} ; \xi, \xi^{\prime}\right)
$$

where it is clear that the integration must be performed with respect to the repeating indices $\xi^{\prime \prime}$. Further, let us introduce the scalar product of two functions, $\phi(\xi)$ and $\psi(\xi)$, as

$$
\langle\phi \mid \psi\rangle=\sum_{\xi} \phi^{*}(\xi) f_{0}(\xi) \psi(\xi)
$$

Then, the matrix element for some "operator" $M$ can be determined as

$$
\langle\phi|M| \psi\rangle=\sum_{\xi} \mathrm{d} \xi^{\prime} \phi^{*}(\xi) M\left(\xi, \xi^{\prime}\right) f_{0}\left(\xi^{\prime}\right) \psi\left(\xi^{\prime}\right)
$$


Let $\phi(\xi)=\left\{\phi_{\mu}(\xi)\right\}$ be the orthogonalized basis of functions with the weight $f_{0}(\xi)$, so that the following condition is satisfied:

$$
\left\langle\phi_{\nu} \mid \phi_{\mu}\right\rangle=\delta_{\nu \mu}, \quad \sum_{\nu}\left|\phi_{\nu}\right\rangle\left\langle\phi_{\nu}\right|=1
$$

where

$$
\phi_{\mu}(\xi)=\phi_{l m n}(\xi)=(l ! m ! n !)^{-1 / 2} \bar{H}_{l}\left(\xi_{x}\right) \bar{H}_{m}\left(\xi_{y}\right) \bar{H}_{n}\left(\xi_{z}\right),
$$

$\bar{H}_{l}(\xi)=2^{-l / 2} H_{l}(\xi / 2), H_{l}(\xi)$ is a Hermite polynomial. Then, each function in the system of equations (4.18), which depends on momentum variables $\xi, \xi^{\prime}$, can be expanded over functions $\phi_{\mu}(\xi)$ in the series:

$$
\begin{aligned}
& \tilde{\Phi}\left(\mathbf{k} ; \xi, \xi^{\prime} ; z\right)=\sum_{\nu, \mu} \phi_{\nu}^{*}(\xi) \tilde{\Phi}_{\nu \mu}(\mathbf{k} ; z) \phi_{\mu}\left(\xi^{\prime}\right) f_{0}\left(\xi^{\prime}\right) \\
& \tilde{\Sigma}\left(\mathbf{k} ; \xi, \xi^{\prime} ; z\right)=\sum_{\nu, \mu} \phi_{\nu}^{*}(\xi) \tilde{\Sigma}_{\nu \mu}(\mathbf{k} ; z) \phi_{\mu}\left(\xi^{\prime}\right) f_{0}\left(\xi^{\prime}\right)
\end{aligned}
$$

where

$$
\begin{aligned}
& \tilde{\Phi}_{\nu \mu}(\mathbf{k} ; z)=\left\langle\phi_{\nu}\left|\tilde{\Phi}\left(\mathbf{k} ; \xi, \xi^{\prime} ; z\right)\right| \phi_{\mu}\right\rangle=\sum_{\xi^{\prime}} \phi_{\nu}^{*}(\xi) f_{0}(\xi) \tilde{\Phi}\left(\mathbf{k} ; \xi, \xi^{\prime} ; z\right) \phi_{\mu}\left(\xi^{\prime}\right) \\
& \tilde{\Sigma}_{\nu \mu}(\mathbf{k} ; z)=\left\langle\phi_{\nu}\left|\tilde{\Sigma}\left(\mathbf{k} ; \xi, \xi^{\prime} ; z\right)\right| \phi_{\mu}\right\rangle=\sum_{\xi^{\prime}} \phi_{\nu}^{*}(\xi) f_{0}(\xi) \tilde{\Sigma}\left(\mathbf{k} ; \xi, \xi^{\prime} ; z\right) \phi_{\mu}\left(\xi^{\prime}\right)
\end{aligned}
$$

Let us substitute expansions (4.23)-(4.26) into equation (4.18). As a result, one obtains:

$$
z \tilde{\Phi}_{\nu \mu}(\mathbf{k} ; z)-\sum_{\gamma} \tilde{\Sigma}_{\nu \gamma}(\mathbf{k} ; z) \tilde{\Phi}_{\gamma \mu}(\mathbf{k} ; z)=-\tilde{\Phi}_{\nu \mu}(\mathbf{k}) .
$$

In actual calculations, a finite number of functions from the set $\phi_{\nu}(\xi)$ is used. Taking into account this fact let us introduce the projection operator $P$ which projects arbitrary functions $\psi(\xi)$ onto a finite set of functions $\phi_{\mu}(\xi)$ :

$$
P=\sum_{\nu=1}^{n}\left|\phi_{\nu}\right\rangle\left\langle\phi_{\nu}\right|=1-Q, \quad P\langle\psi|=\sum_{\nu=1}^{n}\left\langle\psi \mid \phi_{\nu}\right\rangle\left\langle\phi_{\nu}\right| .
$$

Here $n$ denotes a finite number of functions. Then, from (4.27) we obtain a system of equations for a finite set of functions $\phi_{\mu}(\xi)$,

$$
\sum_{\gamma=1}^{n}\left[z \bar{\delta}_{\nu \gamma}-\mathrm{i} \tilde{\Omega}_{\nu \gamma}(\mathbf{k})+\tilde{D}_{\nu \gamma}(\mathbf{k} ; z)\right] \tilde{\Phi}_{\gamma \mu}(\mathbf{k} ; z)=-\tilde{\Phi}_{\nu \mu}(\mathbf{k})
$$

where

$$
\tilde{D}_{\nu \mu}(\mathbf{k} ; z)=\left\langle\phi_{\nu}\left|\tilde{\varphi}(\mathbf{k} ; z)+\tilde{\Sigma}(\mathbf{k} ; z) Q[z-Q \tilde{\Sigma}(\mathbf{k} ; z) Q]^{-1} Q \tilde{\Sigma}(\mathbf{k} ; z)\right| \phi_{\mu}\right\rangle
$$

are generalized hydrodynamic transport kernels and

$$
\mathrm{i} \tilde{\Omega}_{\nu \mu}(\mathbf{k})=\left\langle\phi_{\nu}|\mathrm{i} \tilde{\Omega}(\mathbf{k})| \phi_{\mu}\right\rangle
$$


is a frequency matrix. Note that matrices $\mathrm{i} \tilde{\Omega}(\mathbf{k})$ and $\tilde{\varphi}(\mathbf{k} ; z)$ are defined according to (4.3), (4.4) and (4.11)-(4.14).

Let us find solutions to the system of equations (4.19) in the hydrodynamic region when a set of functions $\phi_{\mu}(\xi)$ presents five moments of a one-particle distribution function:

$$
\begin{gathered}
\phi_{1}(\xi)=1, \quad \phi_{2}(\xi)=\xi_{z}, \quad \phi_{3}(\xi)=\frac{1}{\sqrt{6}}\left(\xi^{2}-3\right), \\
\phi_{4}(\xi)=\xi_{x}, \quad \phi_{5}(\xi)=\xi_{y} .
\end{gathered}
$$

Then, the following relations are fulfilled:

$$
\begin{aligned}
\langle 1| \hat{n}_{\mathbf{k}}(\xi) & =\sum_{\xi} \hat{n}_{\mathbf{k}}(\xi)=\hat{n}_{\mathbf{k}}, \\
\left\langle\xi_{\gamma}\right| \hat{n}_{\mathbf{k}}(\xi) & =\sum_{\xi} \hat{n}_{\mathbf{k}}(\xi) \xi_{\gamma}=\hat{p}_{\mathbf{k}}^{\gamma}, \\
\left\langle 6^{-1 / 2}\left(\xi^{2}-3\right)\right| \hat{n}_{\mathbf{k}}(\xi) & =\sum_{\xi} \hat{n}_{\mathbf{k}}(\xi) 6^{-1 / 2}\left(\xi^{2}-3\right)=\hat{\mathcal{E}}_{\mathbf{k}}^{\mathrm{kin}}-3 \hat{n}_{\mathbf{k}} \beta^{-1}=\hat{h}_{\mathbf{k}}^{\mathrm{kin}},
\end{aligned}
$$

for the Fourier components of densities for the number of particles, momentum and the kinetic part of generalized enthalpy (for the Bose system). Besides that, the microscopic conservation laws for densities of the number of particles and momentum can be written in the form:

$$
\begin{aligned}
\langle 1| \dot{\hat{n}}_{\mathbf{k}}(\xi) & =-\mathrm{i} k_{\gamma} \hat{p}_{\mathbf{k}}^{\gamma} m^{-1}, \\
\left\langle\xi_{\alpha}\right| \dot{\hat{n}}_{\mathbf{k}}(\xi) & =-\mathrm{i} k_{\gamma} \widehat{\mathbf{T}}_{\mathbf{k}}^{\gamma \alpha},
\end{aligned}
$$

where $\widehat{\mathbf{T}}_{\mathbf{k}}^{\gamma \alpha}$ is a Fourier component of the stress tensor.

If we choose the direction of wavevector $\mathbf{k}$ along $o z$-axis, then $\phi_{\nu}(\xi), \nu=1,2,3$ will correspond to longitudinal modes, whereas $\phi_{\nu}(\xi)$ at $\nu=4,5$ they will be related to transverse modes.

From the system of equations (4.29), at $\nu=4,5, \phi_{4}(\xi)=\xi_{x}, \phi_{5}(\xi)=\xi_{y}$, one obtains an equation for the Fourier component of the time correlation function connected with the transverse component of the momentum density $\Phi_{44}(\mathbf{k} ; z)$. From this equation one finds:

$$
\Phi_{44}(\mathbf{k} ; z)=\Phi_{p p}^{\perp}(\mathbf{k} ; z)=-\frac{1}{z+D_{p p}^{\perp}(\mathbf{k} ; z)},
$$

where

$$
\begin{aligned}
\Phi_{p p}^{\perp}(\mathbf{k} ; z) & =\left\langle\xi_{x}\left|\Phi_{n n}\left(\mathbf{k}, \xi, \xi^{\prime} ; z\right)\right| \xi_{x}^{\prime}\right\rangle, \\
D_{p p}^{\perp}(\mathbf{k} ; z) & =D_{p p}^{\perp(\text { kin })}(\mathbf{k} ; z)+D_{p p}^{\perp \text { (int })}(\mathbf{k} ; z), \\
D_{p p}^{\perp(\mathrm{kin})}(\mathbf{k} ; z) & =\left\langle\xi_{x}\left|\varphi_{n n}\left(\mathbf{k}, \xi, \xi^{\prime} ; z\right)\right| \xi_{x}^{\prime}\right\rangle, \\
D_{p p}^{\perp \text { (int) }}(\mathbf{k} ; z) & =\left\langle\xi_{x}\left|\left[\tilde{\Sigma}\left(\mathbf{k}, \xi, \xi^{\prime} ; z\right) Q\left[z-Q \tilde{\Sigma}\left(\mathbf{k}, \xi, \xi^{\prime} ; z\right) Q\right]^{-1} Q \tilde{\Sigma}\left(\mathbf{k}, \xi, \xi^{\prime} ; z\right)\right]_{n n}\right| \xi_{x}^{\prime}\right\rangle, \\
D_{p p}^{\perp}(\mathbf{k} ; z) & =\mathrm{i} k^{2} \eta(\mathbf{k} ; z)(m n)^{-1},
\end{aligned}
$$


where $\eta(\mathbf{k} ; z)$ denotes a generalized coefficient of shear viscosity (for the Bose system). Such a coefficient consists of two main contributions. The first one is $D_{p p}^{\perp(\text { kin })}(\mathbf{k} ; z)$, whereas the second contribution $D_{p p}^{\perp \text { (int) }}(\mathbf{k} ; z)$ describes an interplay of kinetic and hydrodynamic processes. The investigation of the contributions $D_{p p}^{\perp(\mathrm{kin})}(\mathbf{k} ; z)$ and $D_{p p}^{\perp \text { (int) }}(\mathbf{k} ; z)$ in the shear viscosity of the quantum Bose system at the decrease of temperature to $2.17 \mathrm{~K}$ presents a significant interest in connection with the beginning of the superfluid component.

If we put $\nu=1,2,3, \phi_{1}(\xi)=1, \phi_{2}(\xi)=\xi_{z}, \phi_{3}(\xi)-6^{-1 / 2}\left(\xi^{2}-3\right)$ in the system of equation (4.29), then we obtain:

$$
\begin{aligned}
& z \Phi_{n a}(\mathbf{k} ; z)-\mathrm{i} \Omega_{n p}(\mathbf{k}) \Phi_{p a}(\mathbf{k} ; z)=-\Phi_{n a}(\mathbf{k}), \\
& z \Phi_{p a}(\mathbf{k} ; z)-\mathrm{i} \Omega_{p n}(\mathbf{k}) \Phi_{n a}(\mathbf{k} ; z)+D_{p p}^{\|}(\mathbf{k} ; z) \Phi_{p a}(\mathbf{k} ; z)-\Sigma_{p h^{\mathrm{kin}}}(\mathbf{k} ; z) \Phi_{h^{\mathrm{kin}_{a}}}(\mathbf{k} ; z) \\
& -\Sigma_{p h^{\mathrm{int}}}(\mathbf{k} ; z) \Phi_{h^{\mathrm{int}} a}(\mathbf{k} ; z)=-\Phi_{p a}(\mathbf{k}) \\
& z \Phi_{h^{\mathrm{kin}_{a}}}(\mathbf{k} ; z)-\Sigma_{h^{\mathrm{kin}} p}(\mathbf{k} ; z) \Phi_{p a}(\mathbf{k} ; z)+D_{h^{\mathrm{kin}} h^{\mathrm{kin}}}(\mathbf{k} ; z) \Phi_{h^{\mathrm{kin}} a}(\mathbf{k} ; z) \\
& +D_{h^{\mathrm{kin}}} h^{\mathrm{int}}(\mathbf{k} ; z) \Phi_{h^{\mathrm{int}} a}(\mathbf{k} ; z)=-\Phi_{h^{\mathrm{kin}} a}(\mathbf{k}), \\
& z \Phi_{h^{\mathrm{int}} a}(\mathbf{k} ; z)-\Sigma_{h^{\mathrm{int}} p}(\mathbf{k} ; z) \Phi_{p a}(\mathbf{k} ; z)+D_{h^{\mathrm{int}} h^{\mathrm{kin}}}(\mathbf{k} ; z) \Phi_{h^{\mathrm{kin}} a}(\mathbf{k} ; z) \\
& +D_{h^{\mathrm{int}}} h^{\mathrm{int}}(\mathbf{k} ; z) \Phi_{h^{\mathrm{int}} a}(\mathbf{k} ; z)=-\Phi_{h^{\mathrm{int}} a}(\mathbf{k})
\end{aligned}
$$

where $a=\left\{\hat{n}_{\mathbf{k}}, \hat{\mathbf{p}}_{\mathbf{k}}, \hat{h}_{\mathbf{k}}^{\text {kin }}, \hat{h}_{\mathbf{k}}^{\text {int }}\right\}$ and

$$
\begin{aligned}
& \Sigma_{p h^{\mathrm{kin}}}(\mathbf{k} ; z)=\mathrm{i} \Omega_{p h^{\mathrm{kin}}}(\mathbf{k})-D_{p h^{\mathrm{kin}}}(\mathbf{k} ; z) \\
& \Sigma_{p h^{\mathrm{int}}}(\mathbf{k} ; z)=\mathrm{i} \Omega_{p h^{\mathrm{int}}}(\mathbf{k})-D_{p h^{\mathrm{int}}}(\mathbf{k} ; z) \\
& \Sigma_{h^{\mathrm{kin} p} p}(\mathbf{k} ; z)=\mathrm{i} \Omega_{h^{\mathrm{kin}} p}(\mathbf{k})-D_{h^{\mathrm{kin}} p}(\mathbf{k} ; z) \\
& \Sigma_{h^{\mathrm{int}} p}(\mathbf{k} ; z)=\mathrm{i} \Omega_{h^{\mathrm{int}} p}(\mathbf{k})-D_{h^{\mathrm{int}} p}(\mathbf{k} ; z)
\end{aligned}
$$

$\mathrm{i} \Omega_{a b}(\mathbf{k})$ and $D_{a b}(\mathbf{k} ; z)$ are determined according to (4.31), (4.30) and describe the correlation between the viscous, kinetic and potential parts of the generalized enthalpy of the Bose system.

From the system of equations (4.41)-(4.44) one can define the Fourier components of the particle number density correlation functions

$$
\Phi_{n n}(\mathbf{k} ; z)=\Phi_{11}(\mathbf{k} ; z)=\left\langle 1\left|\Phi_{n n}\left(\mathbf{k}, \xi, \xi^{\prime} ; z\right)\right| 1^{\prime}\right\rangle
$$

as well as of the longitudinal component of the momentum density

$$
\Phi_{p p}^{\|}(\mathbf{k} ; z)=\Phi_{22}(\mathbf{k} ; z)=\left\langle\xi_{z}\left|\Phi_{n n}\left(\mathbf{k}, \xi, \xi^{\prime} ; z\right)\right| \xi_{z}^{\prime}\right\rangle
$$

for the kinetic part of generalized enthalpy

$$
\Phi_{h^{\mathrm{kin}}} h^{\mathrm{kin}}(\mathbf{k} ; z)=\Phi_{33}(\mathbf{k} ; z)=\left\langle 6^{-\frac{1}{2}}\left(\xi^{2}-3\right)\left|\Phi_{n n}\left(\mathbf{k}, \xi, \xi^{\prime} ; z\right)\right| 6^{-\frac{1}{2}}\left(\left(\xi^{\prime}\right)^{2}-3\right)\right\rangle
$$

as well as for the potential part of generalized enthalpy $\Phi_{h^{\text {int }}} h^{\text {int }}(\mathbf{k} ; z)$ and cross correlation functions, especially $\Phi_{h^{\mathrm{int}} h^{\mathrm{kin}}}(\mathbf{k} ; z), \Phi_{n h^{\mathrm{kin}}}(\mathbf{k} ; z), \Phi_{n h^{\mathrm{int}}}(\mathbf{k} ; z), \Phi_{p h^{\mathrm{kin}}}(\mathbf{k} ; z)$, 
$\Phi_{p h^{\text {int }}}(\mathbf{k} ; z)$. Solving the system of equation (4.41)-(4.44) at $a=n$, one obtains an expression for the correlation function "density-density" $\Phi_{n n}(\mathbf{k} ; z)$

$$
\Phi_{n n}(\mathbf{k} ; z)=-S_{2}(\mathbf{k})\left[z-\frac{\mathrm{i} \Omega_{n p}(\mathbf{k}) \mathrm{i} \Omega_{p n}(\mathbf{k})}{z+\bar{D}_{p p}^{\|}(\mathbf{k} ; z)}\right]^{-1}
$$

where

$$
\begin{aligned}
& \bar{D}_{p p}^{\|}(\mathbf{k} ; z)=D_{p p}^{\|}(\mathbf{k} ; z)-\bar{\Sigma}_{p h^{\mathrm{kin}}}(\mathbf{k} ; z)\left[z+\bar{D}_{h^{\mathrm{kin}} h^{\mathrm{kin}}}(\mathbf{k} ; z)\right]^{-1} \bar{\Sigma}_{h^{\mathrm{kin}} p}(\mathbf{k} ; z) \\
& -\Sigma_{p h^{\mathrm{int}}}(\mathbf{k} ; z)\left[z+D_{h^{\mathrm{int}} h^{\text {int }}}(\mathbf{k} ; z)\right]^{-1} \Sigma_{h^{\mathrm{int}} p}(\mathbf{k} ; z), \\
& \bar{\Sigma}_{p h^{\mathrm{kin}}}(\mathbf{k} ; z)=\Sigma_{p h^{\mathrm{kin}}}(\mathbf{k} ; z) \\
& -\Sigma_{p h^{\text {int }}}(\mathbf{k} ; z)\left[z+D_{h^{\text {int }}} h^{\text {int }}(\mathbf{k} ; z)\right]^{-1} D_{h^{\text {int }}} h^{\text {kin }}(\mathbf{k} ; z), \\
& \bar{\Sigma}_{h^{\mathrm{kin}} p}(\mathbf{k} ; z)=\Sigma_{h^{\mathrm{kin}} p}(\mathbf{k} ; z)- \\
& -D_{h^{\mathrm{kin}}} h^{\mathrm{int}}(\mathbf{k} ; z)\left[z+D_{h^{\mathrm{int}}} h^{\mathrm{int}}(\mathbf{k} ; z)\right]^{-1} \Sigma_{h^{\mathrm{int}} p}(\mathbf{k} ; z), \\
& \bar{D}_{h^{\mathrm{kin}}} h^{\mathrm{kin}}(\mathbf{k} ; z)=D_{h^{\mathrm{kin}} h^{\mathrm{kin}}}(\mathbf{k} ; z) \\
& -D_{h^{\mathrm{kin}}} h^{\mathrm{int}}(\mathbf{k} ; z)\left[z+D_{h^{\mathrm{int}}} h^{\mathrm{int}}(\mathbf{k} ; z)\right]^{-1} D_{h^{\mathrm{int}} h^{\mathrm{kin}}}(\mathbf{k} ; z) .
\end{aligned}
$$

In expressions (4.47)-(4.50) we can observe an interesting renormalization of the functions $\Sigma_{a b}$ and $D_{a b}$ via the generalized transport kernels for fluctuations of flows of the potential part of enthalpy density. However, $\bar{D}_{p p}^{\|}(\mathbf{k} ; z)$ is connected only with the generalized longitudinal viscosity $\eta^{\|}(\mathbf{k} ; z)$, since the densities of the number of particles $\hat{n}_{\mathbf{k}}$ and momentum $\hat{\mathbf{p}}_{\mathbf{k}}$ are included in the set of variables of an abbreviated description. In our case $\bar{D}_{p p}^{\|}(\mathbf{k} ; z)$ takes into account both thermal and viscous dynamical correlation processes. Excluding from (4.46) the imaginary part $\Phi_{n n}^{\|}(\mathbf{k} ; \omega)$ of the correlation function $\Phi_{n n}(\mathbf{k} ; z)$, one obtains an expression for the dynamical structure factor $S(\mathbf{k} ; \omega)$ in which the contributions of transport kernels corresponding to the kinetic and potential parts of the enthalpy density $\hat{h}_{\mathbf{k}}$ are separated. It is evident that the main contribution of the transport kernel $D_{h^{\text {int }}} h^{\text {int }}(\mathbf{k} ; z)$ to the $S(\mathbf{k} ; \omega)$ for liquids was in the hydrodynamical region (the region of small values of wavevector $\mathbf{k}$ and frequency $\omega)$, whereas $D_{h^{\mathrm{kin}} h^{\mathrm{kin}}}(\mathbf{k} ; z)$ will contribute to the kinetic region (of the order of interatomic distances and small correlation times).

It is natural, that their contributions will significantly differ in both the imperfect Bose gas and Bose liquid. We can expect then that the difference of contributions from $D_{h^{\mathrm{kin}} h^{\mathrm{kin}}}(\mathbf{k} ; z)$ and $D_{h^{\mathrm{int}} h^{\mathrm{int}}}(\mathbf{k} ; z)$ will show itself in the dynamic structure factor of semiquantum Bose system in the region of intermediate values of $\mathbf{k}, \omega[25,26]$. In the region of intermediate values of wavevector $\mathbf{k}$ and frequency $\omega$, it is necessary to take into account all the transport kernels $\Sigma_{p h \text { kin }}(\mathbf{k} ; z), \Sigma_{p h^{\text {int }}}(\mathbf{k} ; z), D_{h^{\mathrm{kin}} h^{\mathrm{kin}}}(\mathbf{k} ; z)$, $D_{h^{\text {int }} h^{\text {kin }}}(\mathbf{k} ; z), D_{h^{\text {int }} h^{\text {int }}}(\mathbf{k} ; z)$. Since it is impossible to perform exact calculations of the above described functions, it is necessary in each separate region to accept models corresponding to the physical processes. Obviously, it is necessary to provide the modelling on the level of generalized transport kernels $\varphi_{n n}\left(\mathbf{k}, \mathbf{p}, \mathbf{p}^{\prime} ; t, t^{\prime}\right)$, $\varphi_{n h}\left(\mathbf{k}, \mathbf{p} ; t, t^{\prime}\right), \varphi_{h n}\left(\mathbf{k}, \mathbf{p}^{\prime} ; t, t^{\prime}\right), \varphi_{h h}\left(\mathbf{k} ; t, t^{\prime}\right)(4.11)-(4.14)$. In the limit $k \rightarrow 0, \omega \rightarrow 0$, the cross correlations between the kinetic and potential flows of energy and shear 
flows become not so important and the system of equations (4.46)-(4.50) gives a spectrum of the collective modes inherent in molecular hydrodynamics [25]. For intermediate values of $\mathbf{k}$ and $\omega$, the spectrum of collective modes can be found from the condition

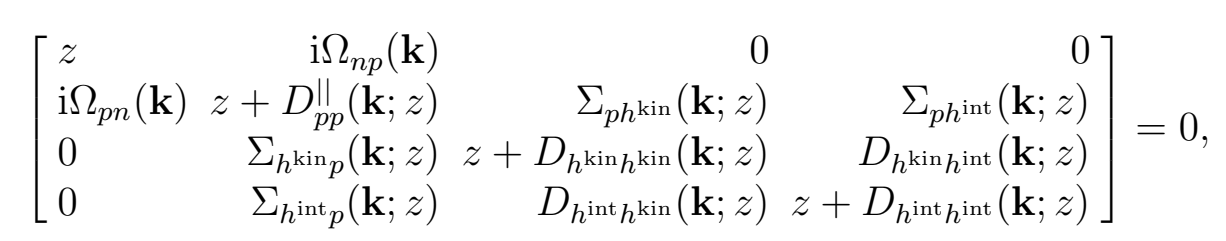

in which the contributions of kinetic and potential parts of generalized enthalpy are separated. This will be reflected in the structure of a heat mode at concrete model calculations of the wavevector- and frequency-dependent transport kernels $D_{h^{\mathrm{kin}} h^{\mathrm{kin}}}(\mathbf{k} ; z), D_{h^{\mathrm{kin}} h^{\mathrm{int}}}(\mathbf{k} ; z), D_{h^{\mathrm{int}} h^{\mathrm{kin}}}(\mathbf{k} ; z), D_{h^{\mathrm{int}} h^{\mathrm{int}}}(\mathbf{k} ; z)$ depending on $\mathbf{k}$ and $\omega$.

The system of equations (4.41)-(4.44) for time correlation functions allows us an extended description of collective modes in the quantum Bose system, which includes both hydrodynamic and kinetic processes. Including some additional functions based on the functions $\phi_{\nu}(\xi)(4.32)$,

$$
\psi_{Q}^{l}(\xi)=\frac{1}{5}\left(\xi^{2}-5\right) \xi_{l}, \quad \psi_{\Pi}^{l j}(\xi)=\frac{\sqrt{2}}{2}\left(\xi_{l} \xi_{j}-\frac{1}{3} \xi^{2} \delta_{l j}\right)
$$

which correspond to a 13-moment approximation of Grad, one obtains a system of equations for time correlation functions of an extended set of hydrodynamic variables $\left\{\hat{n}_{\mathbf{k}}, \hat{\mathbf{p}}_{\mathbf{k}}, \hat{h}_{\mathbf{k}}^{\text {kin }}, \widehat{\boldsymbol{\Pi}}_{\mathbf{k}}, \hat{\mathbf{Q}}_{\mathbf{k}}, \hat{h}_{\mathbf{k}}^{\text {int }}\right\}\left(\widehat{\boldsymbol{\Pi}}_{\mathbf{k}}=\sum_{\xi} \varphi_{\Pi}(\xi) \hat{n}_{\mathbf{k}}(\xi), \hat{\mathbf{Q}}_{\mathbf{k}}=\sum_{\xi} \varphi_{Q}(\xi) \hat{n}_{\mathbf{k}}(\xi)\right):$

$$
\mathrm{i} \tilde{\Omega}^{\mathrm{H}}(\mathbf{k})=\left[\begin{array}{llllll}
0 & \mathrm{i} \Omega_{n p} & 0 & 0 & 0 & 0 \\
\mathrm{i} \Omega_{p n} & 0 & \mathrm{i} \Omega_{p h^{\mathrm{kin}}} & \mathrm{i} \Omega_{p \Pi} & 0 & \mathrm{i} \Omega_{p h^{\mathrm{int}}} \\
0 & \mathrm{i} \Omega_{h^{\mathrm{kin}} p} & 0 & 0 & \mathrm{i} \Omega_{h^{\mathrm{kin}} Q} & 0 \\
0 & \mathrm{i} \Omega_{\Pi p} & 0 & 0 & \mathrm{i} \Omega_{\Pi Q} & 0 \\
0 & 0 & \mathrm{i} \Omega_{Q h^{\mathrm{kin}}} \mathrm{i} \Omega_{Q \Pi} & 0 & \mathrm{i} \Omega_{Q h^{\mathrm{int}}} \\
0 & \mathrm{i} \Omega_{h^{\mathrm{int}} p} & 0 & 0 & \mathrm{i} \Omega_{h^{\mathrm{int}} Q} & 0
\end{array}\right]
$$

is an extended hydrodynamic frequency matrix,

$$
\tilde{\varphi}^{\mathrm{H}}(\mathbf{k} ; z)=\left[\begin{array}{llllll}
0 & 0 & 0 & 0 & 0 & 0 \\
0 & D_{p p}^{H} & D_{p h^{\mathrm{kin}}}^{H} & D_{p \Pi}^{H} & D_{p Q}^{H} & D_{p h^{\mathrm{int}}}^{H} \\
0 & D_{h^{\mathrm{kin}}} & D_{h^{\mathrm{kin}}} h^{\mathrm{kin}} & D_{h^{\mathrm{kin}} \Pi} & D_{h^{\mathrm{kin}}} Q & D_{h^{\mathrm{kin}}} h^{\mathrm{int}} \\
0 & D_{\Pi p} & D_{\Pi h^{\mathrm{kin}}} & D_{\Pi \Pi} & D_{\Pi Q} & D_{\Pi h^{\mathrm{int}}} \\
0 & D_{Q p} & D_{Q h^{\mathrm{kin}}} & D_{Q \Pi} & D_{Q Q} & D_{Q h^{\mathrm{int}}} \\
0 & D_{h^{\mathrm{int}} p} & D_{h^{\mathrm{int}} h^{\mathrm{kin}}} & D_{h^{\mathrm{int}} \Pi} & D_{h^{\mathrm{int}} Q} & D_{h^{\mathrm{int}}} h^{\mathrm{int}}
\end{array}\right]
$$

is a matrix of generalized memory functions, elements of which are transport kernels (4.30) projected based on the functions $\phi_{\nu}(\xi)$ (4.32), (4.52). For such a description, the spectrum of generalized collective modes of the system is determined for intermediate $\mathbf{k}$ and $\omega$ by the relation $\operatorname{det}\left|z \tilde{I}-\mathrm{i} \tilde{\Omega}^{\mathrm{H}}(\mathbf{k})+\tilde{\varphi}^{\mathrm{H}}(\mathbf{k})\right|=0$ which takes into account kinetic and hydrodynamic processes. In the hydrodynamic limit $k \rightarrow 0$, 
$\omega \rightarrow 0$, when the contribution of cross dissipative correlations between the kinetic and hydrodynamic processes practically vanishes, the system of equations for the time correlation function, after some transformations, can be reduced to a system of equations for time correlation functions of densities for the number of particles $\hat{n}_{\mathbf{k}}$, momentum $\hat{\mathbf{p}}_{\mathbf{k}}$, total enthalpy $\hat{h}_{\mathbf{k}}$, the generalized stress tensor $\widehat{\pi}_{\mathbf{k}}=\left(1-\mathcal{P}_{\mathrm{H}}\right) \mathrm{i} L_{N} \hat{\mathbf{p}}_{\mathbf{k}}$ and the generalized enthalpy flow $\hat{\mathbf{q}}_{\mathbf{k}}=\left(1-\mathcal{P}_{\mathrm{H}}\right) \mathrm{i} L_{N} \hat{h}_{\mathbf{k}}$, where $\mathcal{P}_{\mathrm{H}}$ is the Mori operator constructed on the dynamical variables $\left\{\hat{n}_{\mathbf{k}}, \hat{\mathbf{p}}_{\mathbf{k}}, \hat{h}_{\mathbf{k}}\right\}$.

In this case, in the hydrodynamic limit $k \rightarrow 0, \omega \rightarrow 0$ the spectrum of collective excitations coincide with the spectrum $[25,26]$ for semiquantum helium.

However, at fixed values of $\mathbf{k}$ and $\omega$, the transport kernels $\varphi_{\pi \pi}, \varphi_{\pi Q}, \varphi_{Q \pi}, \varphi_{Q Q}$ are expressed via the generalized transport kernels $D_{\nu \mu}(\mathbf{k} ; z)$ of matrix (4.54), i.e. via $\varphi_{n n}\left(\mathbf{k}, \mathbf{p}, \mathbf{p}^{\prime} ; t, t^{\prime}\right), \varphi_{n h}\left(\mathbf{k}, \mathbf{p} ; t, t^{\prime}\right), \varphi_{h n}\left(\mathbf{k}, \mathbf{p}^{\prime} ; t, t^{\prime}\right), \varphi_{h h}\left(\mathbf{k} ; t, t^{\prime}\right)$ (4.11)-(4.14), according to the definition $D_{\nu \mu}(\mathbf{k} ; z)(4.30)$. Here, it is important to point out that passing from the system of transport equations of a self-consistent description of kinetics and hydrodynamics to the equations of generalized hydrodynamics, we can connect the generalized transport kernels (4.11)-(4.14) with the hydrodynamic transport kernels $D_{\nu \mu}(\mathbf{k} ; z)$ in $(4.51)$ or $(4.54)$.

\section{Conclusion}

In this work we have introduced the statistical approach of a consistent description of kinetic and hydrodynamic processes for quantum Bose system far from a point of phase transition. For this purpose we used a method of nonequilibrium statistical operator by D.Zubarev. For parameters of a consistent description, the nonequilibrium one-partial distribution function and average potential energy of interaction Bose particles have been chosen. As a result, the generalized equations of transport (2.33) have been obtained, which in a case of weakly nonequilibrium processes are closed (3.1), (3.2). By means of the latter we have obtained a new system of consistent equations for time correlation functions (4.14)-(4.17) from which the projection method can define a dynamic structure factor, the functions "flow-flow", etc. based on the eigenvectors of nonequilibrium one-partial function. In the hydrodynamical limit this system can be transformed to a system of equations for time correlation functions constructed on the dynamic variables related to the density of particle number, momentum, enthalpy, generalized stress tensor and enthalpy flow for which the spectrum of collective excitations is known [25,26]. However, a special feature here is that the generalized memory functions are constructed on memory functions (2.34)-(2.37) initially obtained in the coupled system of equations for nonequilibrium one-partial distribution functions and average potential energy of interaction. In this way the relation between one-particle and many-particle correlations is traced. It can be actual in a relationship with the studies of the kinetics of one-particle and pair-particle Bose condensate [45,46].

In our approach, a part of the average potential energy of interaction connected with the nonequilibrium distribution function of a pair condensate can be extracted at once. Further we can obtain the system of equations for time correlation func- 
tions with separation of an one-particle and pair-particle Bose condensate both in Hamiltonian [46] and in expressions. Moreover, in our approach we can start with the relevant statistical operator (2.19) to construct a chain of BBGKY equations for nonequilibrium distribution functions of particles with the modified boundary conditions (taking into account multipartial correlations), which is similar both for classical [43] and Fermi systems [47]. This research can be carried out above and the below the points of phase transition with the allocation of condensation distribution functions. In the area of phase transition to a superfluid state of the quantum system, the account of kinetic and hydrodynamical nonlinear fluctuations is important. Here our approach can be modified by choosing as parameters a consistent description of the Wigner nonequilibrium distribution function and nonequilibrium distribution function of collective variables: density of particle number, momentum, energy. In this case the relevant statistical operator can be presented as:

$$
\varrho_{\mathrm{q}}(t)=\exp \left\{-\Phi(t)-\sum_{\mathbf{q} \mathbf{p}} \gamma_{-\mathbf{q}}(\mathbf{p} ; t) \hat{n}_{\mathbf{q}}(\mathbf{p})-\int \mathrm{d} a F(a ; t) \hat{f}(a)\right\},
$$

where $f(a ; t)=\operatorname{Sp} \hat{f}(a) \varrho(t)$ is the nonequilibrium distribution function of collective variables $a_{m \mathbf{k}}: \hat{f}(a)=\delta(\hat{a}-a)=\prod_{m=1}^{3} \prod_{\mathbf{k}} \delta\left(\hat{a}_{m \mathbf{k}}-a_{m \mathbf{k}}\right)$, and $\hat{a}_{1 \mathbf{k}}=\hat{n}_{\mathbf{k}}, \hat{a}_{2 \mathbf{k}}=\hat{\mathbf{P}}_{\mathbf{k}}$, $\hat{a}_{3 \mathbf{k}}=\hat{\varepsilon}_{\mathbf{k}} \cdot f(a ; t)$ satisfies the generalized Fokker-Plank-type equation [38]. These problems will be considered in our subsequent papers.

\section{Acknowledgement}

This study was partially supported by State Foundation for Fundamental Researches, project No. 02.07/418.

\section{References}

1. Bogolubov N.N. To problem on hydrodynamics of superfluid liquid. Preprint JINRR-1395, Dubna, 1963, 49 p. (in Russian).

2. Khalatnikov I.M. The Theory of a Superfluidity. Moscow, Nauka, 1971 (in Russian).

3. Hohenbeig P.C., Martin P.C. // Ann.of Phys., 1965. vol. 34, No. 2, p. 291-359.

4. Krasnikov V.A. // Dokl. Akad. Nauk. SSSR, Ser. Fiz., 1967, vol. 174, No. 5, p. 10371041 (in Russian).

5. Morozov V.G. // Teor. Mat. Fiz., 1976, vol. 28, No. 2, p. 267-280 (in Russian).

6. Peletminsky S.V., Sokolovskii A.I., Chelokov V.S. // Teor. Mat. Fiz., 1978, vol. 34, No. 1, p. 81-98 (in Russian).

7. Patterman S. Superfluid Hydrodynamics. North-Holland Publishing Company, 1974.

8. Skrypnyk V.P., Chelokov V.S. // Teor. Mat. Fiz., 1981. vol. 46, No. 2. p. 242-250 (in Russian).

9. Göze W. Phase Transitions Liquid-Glass. Modern Problems of Physics. Moscow, Nauka, 1992 (in Russian).

10. Tserkovnikov Yu.A. // Teor. Mat. Fiz., 1985, vol. 63, No. 3, p. 440-457 (in Russian). 11. Tserkovnikov Yu.A. // Teor. Mat. Fiz., 1986, vol. 69, No. 3, p. 439-465 (in Russian). 
12. Zubarev D.N., Tserkovnikov Yu.A. Method of Two-Time Temperature Green Functions in Equilibrium and Nonequilibrium Statistical Mechanics. Collection of scientific works of Mathematical Institute of USSR Academy of Sciences. Moscow, Nauka, 1986, vol. 175, No. 3, p. 134-177 (in Russian).

13. Tserkovnikov Yu.A. The Molecular Hydrodynamics of a Quantum Bose Liquid and Method of Two-Time Green Functions. The Modern Problems of a Statistical Physics. Lviv-Kyiv, Naukova dumka, 1989, vol. 1, p. 253-264 (in Russian).

14. Tserkovnikov Yu.A. // Teor. Mat. Fiz., 1990, vol. 85, No. 1, p. 124-149 (in Russian).

15. Tserkovnikov Yu.A. // Teor. Mat. Fiz. 1990. vol. 85, No. 2, p. 258-287 (in Russian).

16. Tserkovnikov Yu.A. // Teor. Mat. Fiz., 1992, vol. 93, No. 3, p. 412-465 (in Russian).

17. Lebedev V.V., Sukhorukov A.I., Khalatnikov I.M. // Zh. Eksp. Teor. Fiz., 1981, vol. 80, p. 1429-1448 (in Russian).

18. Morozov V.G. // Teor. Mat. Fiz., 1986, vol. 67, No. 1, p. 129-142 (in Russian).

19. Morozov V.G. // Physika, 1983, vol. 117A, p. 511-530.

20. Akhiezer A.I., Peletminsky S.V. Methods of Statistical Physics. Moscow, Nauka, 1977 (in Russian).

21. Kirkpatrick T.R., Dorfman I.R. // Journ. of Low Temp. Phys., 1985, vol. 58, No. 3/4. p. 301-331.

22. Glyde H.R. Exitations in Liquid and Solid Helium. Clarendon Press, Oxford, 1994.

23. Glyde H.R., Azuah R.T., Stirling W.G. // Phys. Rev. B., 2000, vol. 62, No. 21, p. $14337-14349$.

24. Szwabinski J., Weypauch M. // Phys. Rev. B., 2001, vol. 64, No. 18, p. 184512-1$184512-16$.

25. Ignatyuk V.V., Mryglod I.M., Tokarchuk M.V. // Low Temp. Phys., 1999, vol. 25, p. 295-302.

26. Ignatyuk V.V., Mryglod I.M., Tokarchuk M.V. // Low Temp. Phys., 1999, vol. 25, p. 857-863.

27. Crevecoeur R.M. ,Verberg R., de Schepper R.M., de Graaf L.A., Montfrooij W. // Phys. Rev. Lett., 1995, vol. 74, p. 5052-5055.

28. Crevecoeur R.M. Analogies in the Microscopic Behavior of Superfluid and Classical Helium Studied by Neutron Scattering. Ph. D. Thesis, Delft, 1996.

29. Crevecoeur R.M., Smorenburg H.E., de Schepper I.M. // J. Low Temp. Phys., 1996, vol. 105 , p. $149-184$.

30. Tserkovnikov Yu.A. // Theor. Math. Phys., 1993. vol.96, No. 3, p.351-372.

31. Bogolubov N.N., Tjablikov S.V. // Dokl. Akad. Nauk. SSSR, Ser. Fiz., 1957. vol. 126, p. 53-56.

32. Zubarev D.N. // Usp. Fiz. Nauk, 1960. vol. 71, No. 1, p. 71-116.

33. Zubarev D.N., Morozov V.G. // Theor. Math. Phys., 1984. vol. 60, No. 2, p. 270-279.

34. Zubarev D.N., Morozov V.G., Omelyan I.P., Tokarchuk M.V. The unification of kinetic and hydrodynamic approaches in the theory of dense gases and liquids. Preprint of the Institute of Theoretical Physics, ITP-88-102R, Kyiv, 1988, 25 p. (in Russian).

35. Zubarev D.N., Morozov V.G., Omelyan I.P., Tokarchuk M.V. // Teor. Mat. Fiz., 1991, vol. 87, No. 1, p. 113-129 (in Russian).

36. Zubarev D.N., Morozov V.G., Omelyan I.P., Tokarchuk M.V. // Teor. Mat. Fiz., 1993, vol. 96, No. 3, p. 325-350 (in Russian).

37. Zubarev D.N. Nonequilibrium Statistical Thermodynamics. New York, Consultant Bureau, 1974. 
38. Zubarev D.N. Modern Methods of Statistical Theory of Nonequilibrium Processes. Reviews of Science and Technology. Modern Problems of Mathematics. Moscow, VINITI, 1980, vol. 15, p. 131-220 (in Russian).

39. Vakarchuk I.O., Hlushak P.A., Tokarchuk M.V. // Ukr. Phys. Journal, 1997, vol. 42, p. $1150-1158$ (in Ukrainian).

40. Kalashnikov V.P. // Teor. Mat. Fiz., 1978. vol. 34, No. 3, p. 412-425 (in Russian).

41. Zubarev D.N., Tokarchuk M.V. // Teor. Mat. Fiz., 1987. vol. 70, No. 2, p. 234-254 (in Russian).

42. Forster D., Martin P.C. Kinetic theory of a weakly coupled fluid. // Phys. Rev. A, 1970, vol. 2, No. 4, p. 1575-1590.

43. Tokarchuk M.V., Omelyan I.P., Kobryn O.E. // Cond. Matt. Phys., 1998, vol. 1, p. $687-751$.

44. Boon J.P., Yip S. Molecular Hydrodynamics. New York, 1980.

45. Vilchinsky S.I., Pashitsky E.A., Fomin P.I. // Low Temp. Phys., 1997, vol. 23, No. 2, p. 1267-1271. (in Russian).

46. Pashitsky E.A. // Low Temp. Phys., 1999, vol. 25, No. 2, p. 81-152.

47. Morozov V.G., Rö̈ke G. // Physica A, 1995, vol. 221, p. 511-538.

\title{
Узгоджений опис кінетики та гідродинаміки квантових бозе-систем
}

\author{
П.А.Глушак, М.В.Токарчук
}

Інститут фізики конденсованих систем НАН України, 79011 Львів, вул. Свєнціцького, 1

Отримано 8 серпня 2004 р.

Запропоновано узгоджений підхід для опису кінетики та гідродинаміки багатобозонних систем. Отримано узагальнені рівняння переносу для сильно і слабо нерівноважних бозе-систем з використанням методу нерівноважного статистичного оператора Д.М. Зубарєва. Отримано нові рівняння для часових кореляційних функцій з виділеними внесками від кінетичної енергії і потенціальної енергій взаємодії частинок. Отримано узагальнені коефіцієнти переносу з урахуванням узгодженого опису кінетичних і гідродинамічних процесів.

Ключові слова: бозе-система, гелій, кінетика, гідродинаміка, кореляційна функція, коефіцієнти переносу

PACS: $67.40 .-w, 47.37 .+q$ 